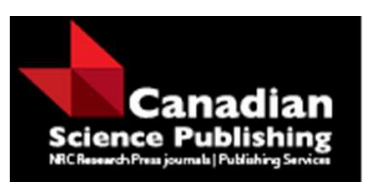

Canadian Journal of Physics

Revue canadienne de physique

\title{
Carrier envelope phase measurement of multi-cycle mid- infrared field and its application on attosecond pulse generation
}

\begin{tabular}{|r|l|}
\hline Journal: & Canadian Journal of Physics \\
\hline Manuscript ID & cjp-2017-0069.R1 \\
\hline Manuscript Type: & Article \\
\hline Date Submitted by the Author: & 18-Aug-2017 \\
\hline Complete List of Authors: & $\begin{array}{l}\text { Feng, Liqiang; Liaoning University of Technology } \\
\text { Li, Wenliang; Xinjiang Institute of Engineering }\end{array}$ \\
\hline Keyword: & $\begin{array}{l}\text { Phase measurement, Multi-cycle mid-infrared field, High-order harmonic } \\
\text { generation, Attosecond pulse generation, Half-cycle harmonic cutoff }\end{array}$ \\
\hline $\begin{array}{r}\text { Is the invited manuscript for } \\
\text { consideration in a Special } \\
\text { Issue? : }\end{array}$ & N/A \\
\hline &
\end{tabular}




\title{
Carrier envelope phase measurement of multi-cycle mid-infrared field and its application on attosecond pulse generation
}

\author{
Liqiang Feng, and Wenliang Li
}

\begin{abstract}
A method for the carrier envelope phase (CEP) measurement of the multi-cycle mid-infrared field has been theoretically presented by using the CEP dependence of the harmonic cutoffs. It shows that by properly adding a CEP-known few-cycle near-infrared pulse to the multi-cycle mid-infrared field, the CEP dependence of the harmonic cutoffs from the combined field become much more distinct in comparison with those from the single mid-infrared field, which provides a route to a new CEP measurement technique. Further, by properly controlling the combined field, three supercontinua of $230 \mathrm{eV}, 242 \mathrm{eV}$ and $266 \mathrm{eV}$ with the less modulation can be obtained. Finally, by selecting the generated harmonics, a series of sub- 35 as pulses in the X-rays region can be produced.
\end{abstract}

Keywords: Carrier envelope phase measurement, Multi-cycle mid-infrared field, High-order harmonic generation, Attosecond pulse generation, Half-cycle harmonic Liqiang Feng. College of Science, Liaoning University of Technology, Jinzhou, 121000, China; State Key Laboratory of Molecular Reaction Dynamics, Dalian Institute of Chemical Physics, Chinese Academy of Sciences, Dalian, 116023, China.

Wenliang Li. Key Laboratory at Universities of Education Department of Xinjiang Uygur Autonomous Region for New Energy Materials, Xinjiang Institute of Engineering, Urumqi, 830091, China.

Corresponding author: Liqiang Feng (e-mail: lqfeng_lngy@126.com). 
cutoff

PACS Nos.: 42.65.Ky, 42.65.Re, 32.80.Fb

\section{INTRODUCTION}

High-order harmonic generation (HHG) has attracted a lot of attention in the past years because the emitted radiation emerges as a broad spectrum with attosecond temporal structure, which may serve as an important tool for accessing the ultrafast scales relevant to electron dynamics in atoms, molecules, solids and materials etc [1-8]. Currently, the HHG in the tunneling regime can be described by the 'three-step' model [9, 10] with the single-active electron approximation. In detail, an electron is first released into continuum by tunneling ionization. Once free, it accelerates and obtains the energy from the laser field. Finally, the ionized electron may recombine with the parent ions and emit the harmonics with energy up to $I_{\mathrm{p}}+3.17 U_{\mathrm{p}}$, where $I_{\mathrm{p}}$ is the ionization potential and $U_{p} \sim I \lambda^{2}$ is the ponderomotive energy of the free electron. Usually, the harmonic emission happens at every half cycle of the laser field and two electronic paths have been shown for a given harmonic order: the short and the long paths $[5,11]$. Therefore, an attosecond pulse train with two main bursts in an optical cycle is formed. But, the single attosecond pulses (SAPs) is much more favorable to the time-resolved dynamics measurement. Thus, several approaches have been proposed to obtain the SAPs, including the ionization and the polarization gating methods [12-20], the attosecond lighthouse [21-23] and the mix-color field method [24-32] etc. Recently, beyond the SAEA, Zhao's group [33, 34], Feng et al. [35] and Koval et al.[36] investigated the HHG 
from the multi-electron models through solving the time-dependent Hartree-Fock equation (TDHF) or the two-electron time-dependent Schrödinger equation (TDSE). They found that due to the multi-electron effect, the harmonic cutoff can be further extended but with a very lower harmonic intensities in the extended region.

As we know the harmonic cutoff frequency $\omega_{\text {cutoff }} \sim U_{\mathrm{p}} \sim I \lambda^{2}$ suggests two schemes to produce the higher photon pulses, namely, (i) to use long wavelengths laser fields, and (ii) to enhance the pulse intensity. Recently, by using the Fourier domain optical parametric amplification, Schmidt et al. [37] produced the 1.8 um mid-infrared field with the pulse duration of two cycles. Popmintchev et al. [38] experimentally showed that by using the mid-infrared laser field, it is possible to extend the maximum harmonic cutoff order and to obtain the higher photon energy. Chou et al. [39] presented an efficient two-color optimal control theory for the mid-infrared harmonic emission and an ultrashort 21 as pulse has been obtained. Li et al. [40] theoretically obtained a SAP of 18 as by using the two-color mid-infrared combined laser field.

Although the extension of the harmonic cutoff can be achieved by using the above schemes, how to control and obtain the carrier envelope phase (CEP)-stable driving pulse is still one of the foremost challenges of the current laser technology. So far, much effort has been devoted to measure and control the phase based on CEP-dependent processes. For instance, (i) based on the CEP-dependent above threshold ionization (ATI) spectra, the single-shot CEP measurement of few-cycle pulses was demonstrated by Wittmann et al. [41]. (ii) Further, this technique was improved to a real-time CEP meter of high precision by using the stereo above-threshold ionization (S-ATI) measurement scheme 
[42-44] and the pulse durations was up to 12.5 fs. (iii) Based on the CEP-dependent high-order harmonic spectra, Harworth et al. [45] measured the CEP of an $8.5 \mathrm{fs} / 800 \mathrm{~nm}$ pulse. Although the CEP measurement can be achieved by using the above methods, the drawback was that the measurements were only possible for ultrashort pulses $(<\sim 12 \mathrm{fs})$.

Thus, in this paper, we propose a promising method to measure the CEP of the multi-cycle mid-infrared field (8 cycle/2000 nm and even 16 cycle/2000 nm, corresponding to the total pulse durations of 55 fs and 110 fs) by introducing a CEP-known few-cycle $800 \mathrm{~nm}$ pulse to a multi-cycle mid-infrared field. It is found that with the introduction of the few-cycle $800 \mathrm{~nm}$ pulse to the multi-cycle mid-infrared field, the harmonic cutoffs depend sensitively on the CEP of the mid-infrared field, which provides a route to measure the CEP of the multi-cycle mid-infrared field. Further, by controlling the combined field with the proper CEPs and the delay times, the harmonic cutoff can be remarkably extended, and three supercontinua with bandwidths of $230 \mathrm{eV}$, $242 \mathrm{eV}$ and $266 \mathrm{eV}$ can be obtained. Finally, by properly selecting particular harmonics orders, a series of attosecond X-ray pulses with the full width at half maximum (FWHM) of sub-35 as can be obtained. Although, the HHG from the two-color [31] or the three-color [32] schemes have been reported in our former investigations, the aims and the results are different. For instance, in Ref. [31], the controlling unipolar pulse has been used to enhance the temporal pulse intensity of the combined field in the half-cycle region and a $406 \mathrm{eV}$ supercontinuum with some sub-35 as pulses can be obtained. In Ref. [32], the controlling $400 \mathrm{~nm}$ and $1600 \mathrm{~nm}$ pulses have been used to control the temporal frequency of the combined field and a $393 \mathrm{eV}$ bandwidth with a 7 as pulse can be 
obtained. The aims of this paper are (i) the measurement of the CEP of the multi-cycle mid-infrared field by using the CEP dependence of the harmonic cutoff and (ii) the extension of the harmonic cutoff and the generation of the SAP by using the multi-cycle mid-infrared combined field.

\section{THEORETICAL METHOD}

The interaction between $\mathrm{He}$ atom and the linear polarization laser field can be investigated by solving the three dimensional TDSE with the single-active electron approximation [46-50] (atomic units are used in this paper unless stated otherwise),

$$
i \frac{\partial \varphi(r, t)}{\partial t}=H(t) \varphi(r, t)=\left[-\frac{1}{2} \nabla^{2}+V(r)-z E(t)\right] \varphi(r, t) .
$$

$V(r)=-1.353 / r$ is the Coulomb potential of He atom. Here, the laser field is linearly polarized along the $z$ axis. Thus the electron motion and the harmonic emission are mainly along the $z$ axis.

The combined field is given by,

$$
\begin{aligned}
& E(t)=E_{1} f_{1}(t) \cos \left(\omega_{2000} t+\varphi_{1}\right)+E_{2} f_{2}\left(t-\tau_{\text {delay } 1}\right) \cos \left(\omega_{800}\left(t-\tau_{\text {delay } 1}\right)+\varphi_{2}\right) \\
& +E_{3} f_{3}\left(t-\tau_{\text {delay } 2}\right) \cos \left(\omega_{2400}\left(t-\tau_{\text {delay } 2}\right)+\varphi_{3}\right)
\end{aligned} .
$$

Here $\omega_{2000}, \omega_{800}$ and $\omega_{2400}$ are the frequencies of the $2000 \mathrm{~nm}, 800 \mathrm{~nm}$ and $2400 \mathrm{~nm}$ laser pulses, respectively. $E_{\mathrm{i}}$ and $\varphi_{\mathrm{i}}(i=1 \sim 3)$ are the amplitudes and the CEPs of the above three pulses. $\tau_{\text {delay1 }}$ and $\tau_{\text {delay } 2}$ are the delay times between the $2000 \mathrm{~nm}$ and $800 \mathrm{~nm}$ and between the $2000 \mathrm{~nm}$ and the $2400 \mathrm{~nm}$ pulses, respectively. The envelope function is,

$$
f_{i}(t)=\exp \left[-2 \ln (2) t^{2} / \tau_{i}^{2}\right]
$$

where, $\tau_{\mathrm{i}}(\mathrm{i}=1 \sim 3)$ are the FWHM of the above three pulses. 
The time-dependent wave function $\phi(r, t)$ can be expanded as $\phi(r, t)=\sum_{l=0}^{l_{\text {max }}=50} \frac{1}{r} \chi_{l}(r, t) Y_{l}^{0}(\theta)$, where $\chi_{l}(r, t)$ and $Y_{l}^{0}(\theta)$ are the radial and the spherical harmonics functions. In the present calculations, the maximum radius of 1000 a.u. with the grid spacing of 0.1 a.u., and maximum number of partial waves of $l_{\max }=50$ is sufficient to obtain converged results. The time step is chosen to be 0.05 a.u..

The HHG spectra are given by,

$$
S(\omega)=\left|\frac{1}{\sqrt{2 \pi}} \int_{0}^{T_{\text {total }}} a(t) e^{-i \omega t} d t\right|^{2},
$$

where, $T_{\text {total }}$ is the total interaction time and $a(t)$ is given by $[51,52]$ $a(t)=-\langle\varphi(r, t)|[H(t),[H(t), r] \rrbracket \varphi(r, t)\rangle$ (much more detail information can be found in Ref. 52).

Time-frequency analyses of the HHG can be obtained by using the wavelet transformation of the dipole acceleration $a(t)$ [53],

$$
A(t, \omega)=\int \mathrm{a}\left(t^{\prime}\right) \sqrt{\omega} W\left(\omega\left(t^{\prime}-t\right)\right) d t^{\prime},
$$

where $W(x)=\left(\frac{1}{\sqrt{\xi}}\right) e^{i x} e^{-x^{2} / 2 \xi^{2}}$ is the Morlet wavelet with $\xi=18$ a.u..

Temporal profiles of the SAPs can be given by,

$$
I(t)=\left|\sum_{q} a_{q} e^{i q \omega t}\right|^{2},
$$

where, $a_{q}=\int \mathrm{a}(t) e^{-i q \omega t} d t$.

\section{RESULTS AND DISCUSSION}




\subsection{CEP dependence of the harmonic cutoffs on the CEP measurement}

Before our discussion, we first define two concepts in this paper. As we know the harmonic emission happens at every half-cycle of the laser pulse. Thus, for each half-cycle, there should be one specified harmonic cutoff. Here, we define the maximum harmonic cutoff from the half-cycle as the MHC and the second maximum harmonic cutoff from the half-cycle as the HHC.

Figure 1(a) and Table 1 show the varieties of the MHCs and the HHCs as a function of the CEP of the single-color $800 \mathrm{~nm}$ field (here, we use $\varphi_{800}$ as the CEP of the $800 \mathrm{~nm}$ pulse for the single-color field). The FWHM and the pulse intensity are 5 fs (corresponding to the total pulse duration of 13.75 fs or 5 cycles) and $I_{800}=$ $5.0 \times 10^{14} \mathrm{~W} / \mathrm{cm}^{2}$ (here, we use $I_{800}$ as the pulse intensity of the $800 \mathrm{~nm}$ pulse for the single-color field). As seen, given a particular couple of the MHC and the $\mathrm{HHC}$, it does exist a unique value of the CEP that matches them, which means the ensemble of harmonic cutoff couple is uniquely defined. However, due to the period of $\pi$ for the CEP dependence of the harmonic cutoffs driven by the single-color few-cycle field, only the laser phases from $\varphi_{800}=0.0 \pi$ to $\varphi_{800}=1.0 \pi$ can be measured by using the single-color 800nm pulse. To clearly see the HHG spectra driven by the different CEPs of the laser field, in Fig. 1(b), we present the HHG spectra driven by the above $800 \mathrm{~nm}$ field with $\varphi_{800}=0.0 \pi$ and $\varphi_{800}=0.5 \pi$. It shows that the MHCs and the HHCs of the two CEPs are $E_{M H C}=81 \omega_{800}, E_{H H C}=55 \omega_{800}$ for $\varphi_{800}=0.0 \pi$ and $E_{M H C}=70 \omega_{800}, E_{H H C}=42 \omega_{800}$ for $\varphi_{800}=$ $0.5 \pi$, respectively. Moreover, there are two harmonic plateaus on the HHG spectra and the second harmonic plateau from $\varphi_{800}=0.5 \pi$ is higher than that from $\varphi_{800}=0.0 \pi$. To 
understand the physical mechanism behind the CEP dependence of the harmonic spectra, in Figs. 1(c)-1(e), we presents the laser profiles, the ionization probabilities (IPs) and the time-frequency analyses of the HHG for the cases of the $5 \mathrm{fs} / 800 \mathrm{~nm}$ pulses with $\varphi_{800}=$ $0.0 \pi$ and $\varphi_{800}=0.5 \pi$ (here to clearly see the laser profiles and the harmonic emission, only the middle part of the laser field and the harmonic emission processes from $t=$ $-2.0 \mathrm{~T}_{800}$ to $t=2.0 \mathrm{~T}_{800}$ are shown, where $\mathrm{T}_{800}$ is the optical cycle of the $800 \mathrm{~nm}$ field). Clearly, four main ionized time near the peaks of the laser fields can be found, i.e., $t=$ $-0.9 \mathrm{~T}_{800}, t=-0.4 \mathrm{~T}_{800}, t=0.1 \mathrm{~T}_{800}, t=0.6 \mathrm{~T}_{800}$ for the case of $\varphi_{800}=0.0 \pi$ and $t=-1.2 \mathrm{~T}_{800}, t$ $=-0.7 \mathrm{~T}_{800}, t=-0.2 \mathrm{~T}_{800}, t=0.3 \mathrm{~T}_{800}$ for the case of $\varphi_{800}=0.5 \pi$. Further, the free electron is accelerated away from the ion and is drawn back to the ion during the following $2 / 3$ optical cycle. As a result, four harmonic radiation peaks (HRPs), marked as $A_{1 \sim 4}$, can be found, as shown in Figs. 1(d) and 1(e). According to the three-step model, the harmonic cutoffs from each HRPs are relevant to $E_{\text {cutoff }} \sim I \lambda^{2}$. For the single-color field, the wavelength is the same for every half cycles, thus, the harmonic cutoffs of each HRPs are only proportional to temporal intensities of the half-cycles. In detail, for the case of $\varphi_{800}=$ $0.0 \pi$ [Fig. 1(d)], when the harmonics are higher than $60 \omega_{800}$, the contribution of the HHG is only from $A_{2}$; while when the harmonics are lower than $60 \omega_{800}$ all the four HRPs are contributed to the HHG, thus leading to the visible two harmonic plateaus on the HHG spectrum. It is noted that due to the lower IPs around the first ionization process, the intensity of $A_{1}$ is much weaker than those of the other three HRPs. For the case of $\varphi_{800}=$ $0.5 \pi$, due to the CEP effect, the temporal intensities of the half cycles (i) from $t=-1.0 \mathrm{~T}_{800}$ to $t=-0.5 \mathrm{~T}_{800}$, from $t=-0.5 \mathrm{~T}_{800}$ to $t=0.0$ and (ii) from $t=0.0$ to $t=0.5 \mathrm{~T}_{800}$, from $t=$ 
$0.5 \mathrm{~T}_{800}$ to $t=1.0 \mathrm{~T}_{800}$ are respectively decreased and increased in comparison with those from $\varphi_{800}=0.0 \pi$ case. As a result, the harmonic cutoffs from (i) $A_{1}, A_{2}$ and (ii) $A_{3}, A_{4}$ are respectively decreased and increased compared with those produced from $\varphi_{800}=0.0 \pi$ case, which is responsible for the CEP dependence of the harmonic cutoffs. Moreover, when the harmonics are higher than $45 \omega_{800}$, there are two HRPs of $A_{2}$ and $A_{3}$ contributed to the HHG, which is the reason behind the higher intensity of the second harmonic plateau for the case of $\varphi_{800}=0.5 \pi$.

Through the above analyses, we see that the CEP of the few-cycle pulse can be measured by using the single-color CEP-dependent harmonic cutoff scheme. However, due to the period of $\pi$ for the CEP dependence of the harmonic cutoffs driven by the single-color few-cycle field, only the CEPs from $0.0 \pi$ to $1.0 \pi$ can be measured for the case of the few-cycle near-infrared field.

Figure 2(a) and Table 2 show the CEP dependence of the MHCs and the HHCs driven by the single-color multi-cycle $2000 \mathrm{~nm}$ field. The FWHM and the pulse intensity are $20 \mathrm{fs}$, corresponding to the total pulse duration of $55 \mathrm{fs}$ (or 8 cycles) and $I_{2000}=$ $5.0 \times 10^{13} \mathrm{~W} / \mathrm{cm}^{2}$. It shows that the MHCs and the HHCs are around the $131 \omega_{2000}$ for all the CEP values, which means the CEP dependence of the harmonic cutoff is invisible for the single-color multi-cycle mid-infrared field. Figure 2(b) and Table 3 show the CEP dependence of the MHCs and the HHCs driven by the above $20 \mathrm{fs} / 2000 \mathrm{~nm}$ field combined with a CEP known few-cycle near-infrared field. The near-infrared field is chosen to be $5 \mathrm{fs} / 800 \mathrm{~nm}$ with $I_{800}=5.0 \times 10^{14} \mathrm{~W} / \mathrm{cm}^{2}, \varphi_{800}=0.0 \pi$ and $\tau_{\text {delay } 1}=0.0$. It shows that with the introduction of the CEP known few-cycle near-infrared field, the 
dependence of the MHCs and HHCs on the CEP of the multi-cycle mid-infrared field can be resolved. Particularly, the CEP dependence of the harmonic cutoffs can be separated into two parts, (i) the high resolution part from the $\varphi_{2000}=0.0 \pi$ to $\varphi_{2000}=0.4 \pi$, from the $\varphi_{2000}=0.9 \pi$ to $\varphi_{2000}=1.2 \pi$ and from the $\varphi_{2000}=1.6 \pi$ to $\varphi_{2000}=2.0 \pi$ with the monotone increasing or decreasing MHCs and HHCs; (ii) the low resolution part from the $\varphi_{2000}=$ $0.5 \pi$ to $\varphi_{2000}=0.8 \pi$ and from the $\varphi_{2000}=1.3 \pi$ to $\varphi_{2000}=1.5 \pi$ with the near-stable MHCs and HHCs. Figures 2(c) and 2(d) show the HHG spectra driven by the single $20 \mathrm{fs} / 2000$ $\mathrm{nm}$ and the above two-color combined fields with $\varphi_{2000}=0.0 \pi$ and $\varphi_{2000}=0.5 \pi$. For the cases of the single-color $2000 \mathrm{~nm}$ fields [Fig. 2(c)], the MHCs are around the $E_{M H C S}=$ $131 \omega_{2000}$ for both cases and the harmonic modulations are very large. For the cases of the two-color combined fields [Fig. 2(d)], the MHCs and HHCs are $E_{M H C}=403 \omega_{2000}, E_{H H C}=$ $152 \omega_{2000}$ and $E_{M H C}=288 \omega_{2000}, E_{H H C}=115 \omega_{2000}$ for the cases of $\varphi_{2000}=0.0 \pi, \varphi_{800}=0.0 \pi$ and $\varphi_{2000}=0.5 \pi, \varphi_{800}=0.0 \pi$, respectively, which is much better for the CEP measurement. Moreover, two obvious harmonic plateaus can be observed for the cases of the combined fields and the less modulation on the second harmonic plateau can be obtained for the case of the combined field with $\varphi_{2000}=0.0 \pi, \varphi_{800}=0.0 \pi$. But, the intensity of the second plateau for the case of $\varphi_{2000}=0.0 \pi, \varphi_{800}=0.0 \pi$ is much lower than that from $\varphi_{2000}=0.5 \pi$, $\varphi_{800}=0.0 \pi$. It is noted that the typical characteristic of the harmonic spectra shows that only the odd harmonics can be generated. However, due to the broken symmetry of the laser field by adding the second pulse, the harmonic spectra become continuous and both the odd and the even harmonics can be found. But, this is not the main aim of the present paper, thus, we do not discuss them in the following discussion. 
Figures 3(a)-3(c) show the laser profiles, the IPs and the time-frequency analyses of the HHG for the cases of the single-color $20 \mathrm{fs} / 2000 \mathrm{~nm}$ pulses with $\varphi_{2000}=0.0 \pi$ and $\varphi_{2000}=0.5 \pi$. Clearly, the numbers of the HRPs caused by the 'three-step' process are increased as the pulse duration increases. Here, we focus on the HRPs with the higher harmonics, marked as $A_{1 \sim 3}$. For the case of $\varphi_{2000}=0.0 \pi$ [Fig. 3(b)], only $A_{2}$ is contributed to the MHC and all three HRPs of $A_{1 \sim 3}$ are contributed to the HHC. However, due to the small difference between the MHC and the HHC (about $5 \omega_{2000}$ ), we cannot observe the obvious two harmonic plateaus on the HHG spectrum. For the case of $\varphi_{2000}=0.5 \pi$ [Fig. 3(c)], we see that the temporal pulse intensities and the IPs from $t=-0.5 \mathrm{~T}_{2000}$ to $t=0.0$ produce almost no change compared with those from $t=-0.25 \mathrm{~T}_{2000}$ to $t=0.25 \mathrm{~T}_{2000}$ in the case of $\varphi_{2000}=0.0 \pi\left(T_{2000}\right.$ is the optical cycle of $2000 \mathrm{~nm}$ pulse). Thus, the accelerated electron will obtain and emit the similar MHC from the HRP of $A_{2}$ in comparison with the case of $\varphi_{2000}=0.0 \pi$. Also, due to the CEP effect, the harmonic cutoffs from $A_{1}$ and $A_{3}$ are decreased and increased, respectively. But, the difference between the MHC and the HHC are also very small. As a result, the CEP dependence of the harmonic cutoff in the single-color multi-cycle field is invisible. Figures 3(d)-3(f) show the laser profiles, the IPs and the time-frequency analyses of the HHG for the cases of the above two-color combined fields with $\varphi_{2000}=0.0 \pi, \varphi_{800}=0.0 \pi$ and $\varphi_{2000}=0.5 \pi, \varphi_{800}=0.0 \pi$. Clearly, with the introduction of the few-cycle $800 \mathrm{~nm}$ pulse, the laser profiles produce a much narrower envelope and a higher laser intensity from $t=-1.0 \mathrm{~T}_{2000}$ to $t=1.0 \mathrm{~T}_{2000}$ as compared to the cases of the single-color $800 \mathrm{~nm}$ or the single-color $2000 \mathrm{~nm}$ fields, which will induce a greater asymmetry in the temporal profiles of the harmonics emitted. 
For instance, the temporal amplitudes (i) from $t=-0.275 \mathrm{~T}_{2000}$ to $t=-0.115 \mathrm{~T}_{2000}$; from $t=$ $-0.115 \mathrm{~T}_{2000}$ to $t=0.1 \mathrm{~T}_{2000}$; from $t=0.325 \mathrm{~T}_{2000}$ to $t=0.925 \mathrm{~T}_{2000}$ and (ii) from $t=0.1 \mathrm{~T}_{2000}$ to $t=0.325 \mathrm{~T}_{2000}$ for the case of $\varphi_{2000}=0.5 \pi, \varphi_{800}=0.0 \pi$ are respectively reduced and enhanced in comparison with the case of $\varphi_{2000}=0.0 \pi, \varphi_{800}=0.0 \pi$. Thus, the acceleration and the recombination events for the ionized electrons will different. Particularly, the harmonic cutoffs from (i) $A_{1}, A_{2}, A_{4}$ and (ii) $A_{3}$ for the case of $\varphi_{2000}=0.5 \pi, \varphi_{800}=0.0 \pi$ can be decreased and increased, respectively, compared with those produced from $\varphi_{2000}=$ $0.0 \pi, \varphi_{800}=0.0 \pi$, which is responsible for the visible CEP dependence of the MHCs and HHCs shown in Fig. 2(d). Moreover, for the case of $\varphi_{2000}=0.0 \pi, \varphi_{800}=0.0 \pi$ [Fig. 3(e)], the contributions of the higher harmonics (higher than $152 \omega_{2000}$ ) are from $A_{1}$ and $A_{2}$. However, the intensity of $A_{1}$ is too weak compared with that of $A_{2}$. Thus, $A_{2}$ plays the main role in the generation of the second harmonic plateau. Moreover, the contribution of the short quantum path for the HRP of $A_{2}$ is higher than that of the long quantum path [11], which is responsible for the less modulation on the second harmonic plateau. For the case of $\varphi_{2000}=0.5 \pi, \varphi_{800}=0.0 \pi$ [Fig. 3(f)], two main HRPs of $A_{2}$ and $A_{3}$ are contributed to the second harmonic plateau (harmonics higher than $115 \omega_{2000}$ ), thus leading to the higher intensity of the second harmonic plateau from the case of $\varphi_{2000}=$ $0.5 \pi, \varphi_{800}=0.0 \pi$

Figure 4(a) shows the effect of the controlling laser intensity on the CEP dependence of the MHCs and the HHCs. The intensity of the few-cycle $5 \mathrm{fs} / 800 \mathrm{~nm}$ pulse is $I_{800}=7.0 \times 10^{14} \mathrm{~W} / \mathrm{cm}^{2}$. Clearly, as the pulse intensity increases, the CEP dependence of the MHCs and the HHCs can also be achieved, but the low resolutions of 
the MHCs and the HHCs from the $\varphi_{2000}=0.5 \pi$ to $\varphi_{2000}=0.8 \pi$ still exist. Figures $4(\mathrm{~b})$ and 4(c) show the CEP dependence of the MHCs and the HHCs driven by the above two-color combined field with $\tau_{\text {delayl }}=0.0, \varphi_{800}=0.5 \pi$ and $\tau_{\text {delayl }}=\mathrm{T}_{800} / 8, \varphi_{800}=0.5 \pi$, respectively. It is found that although the distributions of the MHCs and the HHCs have been changed with the variety of the delay time of $\tau_{\text {delay } 1}$ and the CEP of $\varphi_{800}$, the dependence of the MHCs and the HHCs on the CEP of $\varphi_{2000}$ can also be resolved. Moreover, the MHCs from the $\varphi_{2000}=0.5 \pi$ to $\varphi_{2000}=0.9 \pi$ and the HHCs from the $\varphi_{2000}=$ $1.4 \pi$ to $\varphi_{2000}=1.6 \pi$ produce the monotone increasing characteristic, which is beneficial to improve the resolution of the CEP measurement. Figure 4(d) shows the CEP dependence of the MHCs and the HHCs driven by the two-color field consisting of a $40 \mathrm{fs} / 2000 \mathrm{~nm}$ pulse (corresponding to the total pulse duration of $110 \mathrm{fs}$ ) and a $5 \mathrm{fs} / 800 \mathrm{~nm}$ pulse with $\tau_{\text {delay } 1}=0.0$ and $\varphi_{800}=0.0 \pi$. It shows that the CEP dependence of the MHCs and the HHCs can also be distinguished when the FWHM of the mid-infrared is increased up to $40 \mathrm{fs}$, which is much better for the measurement of the CEP of the mid-infrared with the very long pulse duration.

Through the above analyses, the CEP of the multi-cycle mid-infrared field can be determined and measured by using the two-color CEP-dependent harmonic cutoff scheme. But, it still has some limits, for instance, (i) a few-cycle CEP-known near-infrared pulse is necessary. (ii) The low resolution part of the MHCs and the HHCs for some CEPs is unbeneficial to accurately measure the CEPs of the laser field. (iii) The laser field can yield any signature of the CEP, thus, much larger theoretical data about the CEP dependence of the MHCs and HHCs are needed for predicting the CEPs of the laser 
field.

\subsection{Harmonic cutoff extension and attosecond pulse generation}

As we know except for the above proposed application to measure the CEPs of the laser field, another important application of the HHG is to produce the SAPs, especially for using the mid-infrared field to extending the harmonic cutoff, which is a hot issue in the current strong field physics [37-40, 54]. Thus, in the following part, we show and discuss the HHG and the production of the SAPs by using the combined mid-infrared field.

Figures 5(a)-5(c) show the HHG spectra driven by the three-color combined field consisting of the two-color multi-cycle mid-infrared field and a few-cycle near-infrared pulse with the different CEPs. Here the CEP of one laser field is varied while those of the other two laser fields are kept zero at each manipulation. The laser fields are (i) 20 $\mathrm{fs} / 2000 \mathrm{~nm}, I_{1}=5.0 \times 10^{13} \mathrm{~W} / \mathrm{cm}^{2}$, (ii) $5 \mathrm{fs} / 800 \mathrm{~nm}, I_{2}=5.0 \times 10^{14} \mathrm{~W} / \mathrm{cm}^{2}$ and (iii) 20 $\mathrm{fs} / 2400 \mathrm{~nm}, I_{3}=5.0 \times 10^{13} \mathrm{~W} / \mathrm{cm}^{2}$. It is found that with the respective increase of $\varphi_{1}$ or $\varphi_{3}$, the MHCs are reduced. While with the increase of $\varphi_{2}$, the MHCs are extended. Especially for the case of $\varphi_{2}=1.0 \pi, \varphi_{1}=\varphi_{3}=0.0 \pi$, the HHG presents the visible two harmonic plateaus and a $230 \mathrm{eV}$ supercontinuum from $390 \omega_{2000}$ to $760 \omega_{2000}$ with less harmonic modulation can be obtained.

Figure 6 shows the laser profiles, the IPs and the time-frequency analyses of the HHG for the cases of the above three-color combined fields with the different combinations of the CEPs. Firstly, for the cases of $\varphi_{1}=0.2 \pi, \varphi_{1}=1.0 \pi$ and $\varphi_{3}=0.2 \pi, \varphi_{3}=$ 
$1.0 \pi$, as shown in Figs. 6(a)-6(c) and Figs. 6(g)-6(i), we see that as the CEPs of $\varphi_{1}$ or $\varphi_{3}$ increase, the temporal amplitudes and the IPs from $t=-0.1 \mathrm{~T}_{2000}$ to $t=0.1 \mathrm{~T}_{2000}$ are decreased. As a result, the recombination energy from the maximum HRP of $A_{2}$ is decreased with the increase of $\varphi_{1}$ or $\varphi_{3}$, which is responsible for the decreased cutoffs of the harmonic spectra shown in Figs. 5(a) and 5(c). For the cases of $\varphi_{2}=0.6 \pi$ and $\varphi_{2}=$ $1.0 \pi$, as shown in Figs. 6(d)-6(f), the temporal amplitudes from (i) $t=-0.2 \mathrm{~T}_{2000}$ to $t=0.0$, from $t=0.0$ to $t=0.2 \mathrm{~T}_{2000}$ and (ii) from $t=0.2 \mathrm{~T}_{2000}$ to $t=0.4 \mathrm{~T}_{2000}$ are respectively decreased and increased as the CEP of $\varphi_{2}$ increases. Thus, the recombination energies from the HRPs of (i) $A_{1}, A_{2}$ and (ii) $A_{3}$ are respectively decreased and increased with the increase of $\varphi_{2}$. However, the intensities of $A_{1}$ and $A_{3}$ for $\varphi_{2}=0.6 \pi$ and $A_{1}$ for $\varphi_{2}=1.0 \pi$ are lower than those of the others. Thus, the MHCs are mainly contributed from $A_{2}$ for $\varphi_{2}=$ $0.6 \pi$ and from $A_{3}$ for $\varphi_{2}=1.0 \pi$. As discussed before, the harmonic cutoffs from $A_{2}$ and $A_{3}$ are respectively decreased and increased with the increase of $\varphi_{2}$, thus leading to the extension of the harmonic cutoff as shown in Fig. 5(b). Moreover, when the harmonics are higher than $390 \omega_{2000}$, only $A_{2}$ or $A_{3}$ are contributed to the HHG for the cases of $\varphi_{2}=$ $0.6 \pi$ or $\varphi_{2}=1.0 \pi$, while when the harmonics are lower than $390 \omega_{2000}$, the contributions of the HHG are from $A_{2}$ and $A_{4}$ for $\varphi_{2}=0.6 \pi$ or from $A_{2}, A_{3}$ and $A_{4}$ for $\varphi_{2}=1.0 \pi$, thus leading to the visible two harmonic plateaus on the HHG spectra. Moreover, for the case of $\varphi_{2}=1.0 \pi$, the contribution of the short quantum path for the HRP of $A_{3}$ is higher than that of the long quantum path [11], which is responsible for the smaller interference on the second harmonic plateau.

Figures 7(a) and 7(b) show the HHG spectra driven by the above three-color 
combined fields with the different delay time of $\tau_{\text {delay } 1}$. The delay time of $\tau_{\text {delay } 2}$ is chosen to be $\tau_{\text {delay } 2}=0.0$. The CEPs are chosen to be $\varphi_{1}=0.0 \pi, \varphi_{2}=1.0 \pi, \varphi_{3}=0.0 \pi$. Clearly, as the delay time of $\tau_{\text {delay1 }}$ positive increases [Fig. 7(a)], the MHCs are reduced and the second harmonic plateau is disappeared. While as the delay time of $\tau_{\text {delay1 negative }}$ increases [Fig. 7(b)], the MHCs can be extended but with the larger harmonic modulation, which is unbeneficial to the productions of the SAPs. Thus, through our calculation, $\tau_{\text {delay } 1}=-0.05 \mathrm{~T}_{800}$ is the chosen to be the optimal delay time of $\tau_{\text {delay } 1}$ for the extension of the harmonic cutoff and a $242 \mathrm{eV}$ bandwidth from $400 \omega_{2000}$ to $790 \omega_{2000}$ with the intensity enhancement of 1 order of magnitude can be obtained compared with the $\tau_{\text {delay } 1}=0.0$ case, as shown in Fig. 7(c) for the cases of the original harmonic intensities.

Figure 8 shows the laser profiles, the IPs and the time-frequency analyses of the HHG for the cases of the above three-color combined fields with the different delay time of $\tau_{\text {delay } 1}$. As seen, for the case of $\tau_{\text {delay } 1}=0.15 \mathrm{~T}_{800}$, the temporal amplitudes (i) from $t=$ $-0.2 \mathrm{~T}_{2000}$ to $t=0.0$, from $t=0.0$ to $t=0.2 \mathrm{~T}_{2000}$ and (ii) from $t=0.2 \mathrm{~T}_{2000}$ to $t=0.4 \mathrm{~T}_{2000}$ are respectively enhanced and reduced in comparison with the case of $\tau_{\text {delayl }}=0.0$ [Fig. 8(a)]. Therefore, the recombination energies from the HRPs of (i) $A_{1}, A_{2}$ and (ii) $A_{3}$ are extended and reduced, respectively, compared with the $\tau_{\text {delay } 1}=0.0$ case [Fig. 8(b)]. However, the intensity of $A_{1}$ is too weak, and its contribution to the HHG spectrum can be ignored. As a result, the contributions of the higher harmonics are mainly from $A_{2}$ and $A_{3}$, but the extended harmonic from $A_{2}$ is still lower than that from $A_{3}$ in case of $\tau_{\text {delayl }}=$ 0.0 [see Fig. 6(f)], thus leading to the decrease of the MHCs with the positive increase of $\tau_{\text {delayl }}$. Moreover, due to the decreased differences between the maximum HRP of $A_{2}$ (or 
$\mathrm{A}_{3}$ ) and the second maximum HRP of $A_{4}$, the differences between the MHCs and the HHCs are decreased, which is responsible for the reduced second harmonic plateau with the positive increase of $\tau_{\text {delay1}}$. For the case of $\tau_{\text {delay } 1}=-0.05 \mathrm{~T}_{800}$, the temporal amplitude from $t=0.2 \mathrm{~T}_{2000}$ to $t=0.4 \mathrm{~T}_{2000}$ is slightly enhanced in comparison with the case of $\tau_{\text {delay } 1}$ $=0.0$ [Fig. 8(c)]. Thus, the HRP of $A_{3}$ is slightly extended [Fig. 8(d)] and is responsible for the slight extension of the MHCs with the negative increase of $\tau_{\text {delayl }}$. With the further negative increase of $\tau_{\text {delayl }}$, the temporal amplitude from $t=0.2 \mathrm{~T}_{2000}$ to $t=0.4 \mathrm{~T}_{2000}$ can be further enhanced [Fig. 8(e) for $\tau_{\text {delay } 1}=-0.25 \mathrm{~T}_{800}$ ], thus leading to the further extension of the HRP of $A_{3}$. However, due to the enhanced IPs in the negative delay time, the short and the long quantum paths present the similar contributions to the HRP of $A_{3}$ [Fig. 8(f)], thus resulting in the enhancement of the harmonic modulation on the second harmonic plateau.

Figures 9(a) and 9(b) show the HHG spectra driven by the above three-color combined fields with the different delay time of $\tau_{\text {delay } 2}$. The delay time of $\tau_{\text {delay } 1}$ is chosen to be $\tau_{\text {delay } 1}=-0.05 \mathrm{~T}_{800}$. The CEPs are chosen to be $\varphi_{1}=0.0 \pi, \varphi_{2}=1.0 \pi$ and $\varphi_{3}=0.0 \pi$. It shows that the maximum cutoff of the harmonic spectrum is achieved when $\tau_{\text {delay } 2}=$ $0.05 \mathrm{~T}_{800}$ and a supercontinuum with the bandwidth of $266 \mathrm{eV}$ from $390 \omega_{2000}$ to $820 \omega_{2000}$ can be found. With the positive increase of the delay time of $\tau_{\text {delay2 }}$ [Fig. 9(a)], the modulation on the second harmonic plateau can be increased. With the negative increase of the delay time of $\tau_{\text {delay2 }}$ [Fig. $9(\mathrm{~b})$ ], the MHCs move toward the lower harmonic orders and the second harmonic plateau is disappeared. Moreover, through analyzing the original intensities of the HHG for the combined fields with $\tau_{\text {delay } 2}=0.0$ and $\tau_{\text {delay } 2}=$ 
$0.05 \mathrm{~T}_{800}$ as shown in Fig. 9(c), the harmonic intensity from the $\tau_{\text {delay } 2}=0.05 \mathrm{~T}_{800}$ is 1 order of magnitude higher than that from the $\tau_{\text {delay } 2}=0.0$ case.

Figure 10 shows the laser profiles, the IPs and the time-frequency analyses of the HHG for the cases of the above three-color combined fields with the different delay time of $\tau_{\text {delay } 2}$. For the case of $\tau_{\text {delay } 2}=0.05 \mathrm{~T}_{800}$, the temporal amplitude from $t=0.2 \mathrm{~T}_{2000}$ to $t=$ $0.4 \mathrm{~T}_{2000}$ is slightly enhanced in comparison with the case of $\tau_{\text {delay } 2}=0.0$ [Fig. $\left.10(\mathrm{a})\right]$. As a consequence, the HRP of $A_{3}$ is slightly extended [Fig. 10(b)] and is responsible for the slight extensions of the MHCs shown in Fig. 9(a). With the further positive increase of $\tau_{\text {delay2 }}$, as shown in Fig. 10 (c) for the case of $\tau_{\text {delay } 2}=0.25 \mathrm{~T}_{800}$, the IPs around $t=0.2 \mathrm{~T}_{2000}$ is remarkably enhanced. Therefore, the intensity of the HRP of $A_{3}$ is enhanced and both the short and the long quantum paths contribute to its emission [Fig. 10(d)], which is the reason behind the larger modulation on the second harmonic plateau. For the case of $\tau_{\text {delay } 2}=-0.25 \mathrm{~T}_{800}$, the temporal amplitude from $t=0.2 \mathrm{~T}_{2000}$ to $t=0.4 \mathrm{~T}_{2000}$ is decreased in comparison with the case of $\tau_{\text {delay } 2}=0.0$, thus the HRP of $A_{3}$ is reduced. However, due to the decreased IPs, the intensity of $A_{3}$ is also decreased. As a consequence, the intensities of the harmonics higher than $390 \omega_{2000}$ are very weak and even can be ignored on the HHG spectrum, which is the reason behind the decreased MHCs and the disappeared second harmonic plateau shown in Fig. 9(b).

Figure 11 shows the temporal profiles of the SAPs through the Fourier transformation of the selected harmonics. (i) Firstly, by superposing the total harmonics of the combined field with $\varphi_{1}=0.0 \pi, \varphi_{2}=1.0 \pi, \varphi_{3}=0.0 \pi, \tau_{\text {delay } 1}=0.0, \tau_{\text {delay } 2}=0.0$ [Fig. 5(b)], a 160 as pulse with accompanying satellite pulses can be obtained, as shown in the 
inset of Fig. 11(a). This is attributed to the multi-HRPs during the harmonic emission process [see Fig. 6(f)], the harmonics do not emit from the same HRP. Thus, by superposing these harmonics, the multi-satellite pulses can be obtained. Thus, by selecting the harmonics generated from the single HRP is much better for the generations of the SAPs. Through analyzing the time-frequency analysis of the HHG shown in Fig. 6(f), we see that when harmonics are higher than $390 \omega_{2000}$, only one intense HRP of $A_{3}$ is responsible for the HHG spectrum, and the contribution of the short quantum path is higher than that of the long quantum path, which is better for the generations of the SAPs. Thus, by superposing the harmonics of the above combined field [Fig. 5(b)] from the $390 \omega_{2000}$ to the $490 \omega_{2000}$, from the $490 \omega_{2000}$ to the $590 \omega_{2000}$, from the $590 \omega_{2000}$ to the $690 \omega_{2000}$ and from the $690 \omega_{2000}$ to the $760 \omega_{2000}$, four attosecond X-ray pulses with the FWHM of 30 as, 30 as, 30 as, 34 as can be obtained, as shown in Fig. 11(a). (ii) Secondly, through analyzing the time-frequency analysis of the harmonics driven by the combined field with $\varphi_{1}=0.0 \pi, \varphi_{2}=1.0 \pi, \varphi_{3}=0.0 \pi, \tau_{\text {delay } 1}=-0.05 \mathrm{~T}_{800}, \tau_{\text {delay } 2}=0.0$ as shown in Fig. 8(d), the harmonics higher than $400 \omega_{2000}$ are generated by one intense HRP of $A_{3}$ and the short quantum path produces the main contribution to this HRP. Therefore, by superposing the harmonics of this combined field [Fig. 7(c)] from the $400 \omega_{2000}$ to the $500 \omega_{2000}$, from the $500 \omega_{2000}$ to the $600 \omega_{2000}$, from the $600 \omega_{2000}$ to the $700 \omega_{2000}$ and from the $700 \omega_{2000}$ to the $790 \omega_{2000}$, four attosecond X-ray pulses as short as 32 as, 30 as, 30 as, 34 as have been produced, as shown in Fig. 11(b). (iii) Thirdly, through analyzing the time-frequency analysis of the HHG for the case of the $\varphi_{1}=0.0 \pi, \varphi_{2}=1.0 \pi, \varphi_{3}=0.0 \pi$, $\tau_{\text {delay } 1}=-0.05 \mathrm{~T}_{800}, \tau_{\text {delay } 2}=0.05 \mathrm{~T}_{800}$, as shown in Fig. $10(\mathrm{~b})$, the harmonics higher than 
$390 \omega_{2000}$ are only contributed by the HRP of $A_{3}$. Therefore, by superposing the harmonics of this combined field [Fig. 9(c)] from the $390 \omega_{2000}$ to the $490 \omega_{2000}$, from the $490 \omega_{2000}$ to the $590 \omega_{2000}$, from the $590 \omega_{2000}$ to the $690 \omega_{2000}$ and from the $690 \omega_{2000}$ to the $790 \omega_{2000}$, a 30 as, a 30 as, a 30 as and a 35 as pulses can be produced, as shown in Fig. 11(c). Here, although the short and the long quantum paths merge in one around the harmonic cutoff region, the comparable intensities from both paths are not favorable to produce the SAPs. Thus, there is a sub-pulse showing up around the main pulse when superposing the higher harmonics around the cutoff region. Moreover, the SAPs generated from the case (iii) are almost 2 and 1 orders of magnitude higher than those produced from case (i) and case (ii).

\section{CONCLUSION}

In conclusion, we theoretically propose an efficient method to measure the CEP of the multi-cycle mid-infrared field and to produce the ultrashort SAPs. It is found that:

(1) By adding a CEP-known few-cycle near-infrared pulse as a 'detector' to the multi-cycle mid-infrared pulse, the resolution of the phase-dependence of the MHCs and the HHCs can be significantly improved in comparison with the single multi-cycle mid-infrared pulse case. As a result, by measuring the CEP dependence of the MHCs and the HHCs of the combined field, the CEP of the multi-cycle mid-infrared pulse can be determined. However, the proposed scheme still has some limits, for instance, (i) a few-cycle CEP-known near-infrared pulse is necessary. (ii) The low resolution part of the MHCs and the HHCs for some CEPs can be found. (iii) The much larger theoretical data 
about the CEP depenent MHCs and HHCs are needed for predicting the CEPs of the laser field.

(2) Further, by properly using the three-color field scheme, the harmonic cutoff can be remarkably extended and three bandwidths of $230 \mathrm{eV}, 242 \mathrm{eV}$ and $266 \mathrm{eV}$ with less harmonic modulation can be obtained. Finally, by properly selecting particular harmonics, a series of attosecond X-ray pulses with the FWHM of sub-35 as can be obtained.

\section{ACKNOWLEDGMENTS}

The authors thank Prof. Keli Han and Dr. Ruifeng Lu for providing the computational code used in the present work. We also gratefully acknowledge useful discussions with Prof. Tianshu Chu. This work was supported by National Natural Science Foundation of China (No. 11504151) and Doctoral scientific research foundation of Liaoning province (No. 201501123).

\section{REFERENCES}

[1] F. Krausz and M. Ivanov, Rev. Mod. Phys. 81, 163 (2009).

[2] K. J. Yuan and A. D. Bandrauk, Phys. Rev. Lett. 110, 023003 (2013).

[3] M. Uiberacker, T. Uphues, M. Schultze, A. J. Verhoef, V. Yakovlev, M. F. Kling, J. Rauschenberger, N. M. Kabachnik, H. Schröder, M. Lezius, K. L. Kompa, H. G. Muller, M. J. J. Vrakking, S. Hendel, U. Kleineberg, U. Heinzmann, M. Drescher and F. Krausz, Nature 446, 627 (2007).

[4] E. Goulielmakis, M. Schultze, M. Hofstetter, V. S. Yakovlev, J. Gagnon, M. 
Uiberacker, A. L. Aquila, E. M. Gullikson, D. T. Attwood, R. Kienberger, F. Krausz and U. Kleineberg, Science 320, 1614 (2008).

[5] L. Q. Feng and H. Liu, Can. J. Phys. 94, 651 (2016).

[6] X. B. Bian and A. D. Bandrauk, Phys. Rev. Lett. 113, 193901 (2014).

[7] T. T. Luu, M. Garg, S. Yu. Kruchinin, A. Moulet, M. Th. Hassan and E. Goulielmakis, Nature 521, 498 (2015).

[8] G. Vampa, T. J. Hammond, N. Thiré, B. E. Schmidt, F. Légaré, C. R. McDonald, T. Brabec and P. B. Corkum, Nature 522, 462 (2015).

[9] P. B. Corkum, Phys. Rev. Lett. 71, 1994 (1993).

[10] K. Kulander, K. Schafer and K. L. Krause, NATO Advanced Study Institute, Series B 316, 95 (1993).

[11] Y. Mairesse, A. D. Bohan, L. J. Frasinski, et al., Science 302, 1540 (2003).

[12] F. Ferrari, F.Calegari, M. Lucchini, C. Vozzi, S. Stagira, G. Sansone and M. Nisoli, Nat. Photonics 4, 875 (2010).

[13] M. Louiy, C. L. Arnold, M. Miranda, et al., Optica 2, 563 (2015).

[14] C. M. Heyl, H. Coudert-Alteirac, M. Miranda, et al., Optica 3, 75 (2016).

[15] G. Sansone, E. Benedetti, F. Calegari, et al., Science 314, 443 (2006).

[16] M. Nisoli and G. Sansone, Progress in Quantum Electronics 33, 17 (2009).

[17] P. B. Corkum, N. H. Burnett and M. Y. Ivanov, Opt. Lett. 19, 1870 (1994).

[18] Z. Chang, Phys. Rev. A 71, 023813 (2005).

[19] H. C. Du and B. T. Hu, Opt. Express 18, 25958 (2010).

[20] Q. B. Zhang, P. X. Lu, W. Y. Hong, Q. Liao and S. Y. Wang, Phys. Rev. A, 80, 
033405 (2009).

[21] Vincenti et al., Phys. Rev. Lett 108, 113904 (2012).

[22] C. M. Zhang, G. Vampa, D. M. Villeneuve and P. B. Corkum, J. Phys. B: At. Mol. Opt. Phys. 48, 061001 (2015).

[23] T. J. Hammond, G. G. Brown, K. T. Kim, D.M. Villeneuve and P. B. Corkum, Nature Photonics 10, 171 (2016).

[24] Z. Zeng, Y. Cheng, X. Song, R. Li and Z. Xu, Phys. Rev. Lett. 98, 203901 (2007).

[25] P. F. Lan, P. X. Lu, W. Cao, Y. H. Li and X. L. Wang, Phys. Rev. A 76, 011402 (2007).

[26] X. Cao, S. C.Jiang, C. Yu, Y. H. Wang, L. H. Bai and R. F. Lu, Opt. Express 22, 26153 (2014).

[27] R. F. Lu, H. X. He, Y. H. Guo and K. L. Han, J. Phys. B: At. Mol. Opt. Phys. 42, 225601 (2009).

[28] L. Q. Feng and T. S. Chu, Phys. Rev. A 84, 053853 (2011).

[29] L. Q. Feng and T. S. Chu, J. Chem. Phys. 136, 054102 (2012).

[30] J. Wu, G. T. Zhang, C. L. Xia and X. S. Liu, Phys. Rev. A 82, 013411 (2012).

[31] L. Q. Feng and T. S. Chu, Phys. Lett. A 375, 3641 (2011).

[32] L. Q. Feng and H. Liu, Opt. Commun. 348, 1 (2015).

[33] Z. X. Zhao and J. M. Yuan, Phys. Rev. A 89, 023404 (2014).

[34] B. Zhang, J. M. Yuan and Z. X. Zhao, Phys. Rev. Lett. 111, 163001 (2013).

[35] L. Q. Feng and H. Liu, Chin. Phys. B 24, 034206 (2015).

[36] P. Koval, F. Wilken, D. Bauer and C. H. Keitel, Phys. Rev. Lett. 98, 043904 (2007). 
[37] B. E. Schmidt, N. Thiré, M. Boivin, et al., Nat. Commun. 5, 3643 (2014).

[38] T. Popmintchev, M. C. Chen, O. Cohen, M. Grisham, J. Rocca, M. Murnane and H. Kapteyn, Opt. Lett. 33, 2128 (2008).

[39] Y. Chou, P. C. Li, T. S. Ho and S. I. Chu, Phys. Rev. A 91, 063408 (2015).

[40] P. C. Li, C. Laughlin and S. I. Chu, Phys. Rev. A 89, 023431 (2014).

[41] T. Wittmann, B. Horvath, W. Helml, M. G. Schatzel, X. Gu, A. L. Cavalieri, G. G. Paulus and R. Kienberger, Nat. Phys. 5, 357 (2009).

[42] A. M. Sayler, Tim Rathje, Walter Müller, Klaus Rühle, R. Kienberger and G. G. Paulus, Opt. Lett. 36, 1 (2011).

[43] D. Adolph, A. M. Sayler, T. Rathje, K. Rühle and G. G. Paulus, Opt. Lett. 36, 3639 (2011).

[44] M. Möller, A. M. Sayler, T. Rathje, M. Chini, Zenghu Chang and G. G. Paulus, Appl. Phys. Lett. 99, 121108 (2011).

[45] C. A. Haworth, L. E. Chipperfield, J. S. Robinson, et al., Nat. Phys. 3, 52 (2007).

[46] L. Q. Feng and H. Liu, International Journal of Modern Physics B 29, 1550170 (2015).

[47] R. F. Lu, P. Y. Zhang and K. L. Han, Phys. Rev. E 77, 066701 (2008).

[48] J. Hu, K. L. Han and G. Z. He, Phys. Rev. Lett. 95, 123001 (2005).

[49] Y. C. Han and L. B. Madsen, Phys. Rev. A 81, 063430 (2010).

[50] H. Liu, and L. Q. Feng, Opt. Quant. Electron. 47, 2577 (2015).

[51] K. Burnett, V. C. Reed, J. Cooper and P. L. Knight, Phys. Rev. A 45, 3347 (1992).

[52] L. Q. Feng, Y. B. Duan and T. S. Chu, Ann. Phys. (Berlin) 525, 915 (2013). 
[53] P. Antoine, B. Piraux and A. Maquet, Phys. Rev. A 51, R1750 (1995).

[54] F. Silva, S. M. Teichmann, S. L. Cousin, M. Hemmer and J. Biegert, Nat. Commun. 6, $6611(2015)$. 


\section{Figure Captions}

Fig. 1 (a) The CEP dependence of the MHCs and the HHCs driven by the single-color $800 \mathrm{~nm}$ field. The FWHM and the laser intensity is $5 \mathrm{fs}$ and $I_{800}=5.0 \times 10^{14} \mathrm{~W} / \mathrm{cm}^{2}$. (b) The HHG spectra from the above single-color $5 \mathrm{fs} / 800 \mathrm{~nm}$ fields with $\varphi_{800}=0.0 \pi$ and $\varphi_{800}=$ $0.5 \pi$. (c) Laser profiles and the IPs of the single-color $5 \mathrm{fs} / 800 \mathrm{~nm}$ fields with $\varphi_{800}=0.0 \pi$ and $\varphi_{800}=0.5 \pi$. The time-frequency analyses of the HHG for the cases of the single-color $5 \mathrm{fs} / 800 \mathrm{~nm}$ fields with (d) $\varphi_{800}=0.0 \pi$ and (e) $\varphi_{800}=0.5 \pi$. T 800 is the optical cycle of the $800 \mathrm{~nm}$ pulse.

Fig. 2 The CEP dependence of the MHCs and the HHCs driven by (a) the single-color 20 $\mathrm{fs} / 2000 \mathrm{~nm}$ field and (b) the combined field consisting of the $20 \mathrm{fs} / 2000 \mathrm{~nm}$ field and the $5 \mathrm{fS} / 800 \mathrm{~nm}$ field with $\tau_{\text {delay } 1}=0.0, \varphi_{800}=0.0 \pi$. The pulse intensities are $I_{2000}=$ $5.0 \times 10^{13} \mathrm{~W} / \mathrm{cm}^{2}$ and $I_{800}=5.0 \times 10^{14} \mathrm{~W} / \mathrm{cm}^{2}$, respectively. The HHG spectra from (c) the single-color $20 \mathrm{fs} / 2000 \mathrm{~nm}$ fields with $\varphi_{2000}=0.0 \pi$ and $\varphi_{2000}=0.5 \pi$; and (d) the combined fields with $\varphi_{2000}=0.0 \pi, \varphi_{800}=0.0 \pi$ and $\varphi_{2000}=0.5 \pi, \varphi_{800}=0.0 \pi$.

Fig. 3 Laser profiles of (a) the single-color $20 \mathrm{fs} / 2000 \mathrm{~nm}$ fields with $\varphi_{2000}=0.0 \pi$ and $\varphi_{2000}=0.5 \pi$ and (d) the combined fields with $\varphi_{2000}=0.0 \pi, \varphi_{800}=0.0 \pi$ and $\varphi_{2000}=0.5 \pi$, $\varphi_{800}=0.0 \pi$, respectively. The time-frequency analyses of the HHG for the cases of the single-color $20 \mathrm{fs} / 2000 \mathrm{~nm}$ fields with (b) $\varphi_{2000}=0.0 \pi$ and (c) $\varphi_{2000}=0.5 \pi$ and the combined fields with (e) $\varphi_{2000}=0.0 \pi, \varphi_{800}=0.0 \pi$ and (f) $\varphi_{2000}=0.5 \pi, \varphi_{800}=0.0 \pi . T_{2000}$ means the optical cycle of the $2000 \mathrm{~nm}$ pulse.

Fig. 4 The CEP dependence of the MHCs and the HHCs of the two-color combined field with (a) $I_{800}=7.0 \times 10^{14} \mathrm{~W} / \mathrm{cm}^{2}$; (b) $\tau_{\text {delay } 1}=0.0, \varphi_{800}=0.5 \pi$; (c) $\tau_{\text {delay } 1}=\mathrm{T}_{800} / 8, \varphi_{800}=0.5 \pi$; 
(d) $\tau_{2000}=40 \mathrm{fs}$. The other parameters are the same as those in Fig. 2(b) except for the above given parameters.

Fig. 5 The HHG spectra driven by the three-color combined fields with different CEPs (a) $\varphi_{1}$; (b) $\varphi_{2}$; and (c) $\varphi_{3}$. Here the CEP of one laser field is varied while those of the other two laser fields are kept zero at each manipulation. The laser fields are chosen to be 20 $\mathrm{fs} / 2000 \mathrm{~nm}, I_{1}=5.0 \times 10^{13} \mathrm{~W} / \mathrm{cm}^{2}, 5 \mathrm{fs} / 800 \mathrm{~nm}, I_{2}=5.0 \times 10^{14} \mathrm{~W} / \mathrm{cm}^{2}$ and $20 \mathrm{fs} / 2400 \mathrm{~nm}, I_{3}$ $=5.0 \times 10^{13} \mathrm{~W} / \mathrm{cm}^{2}$. The harmonic intensities have been multiplied by factors of $1,10^{-3}$, $10^{-6}$, and so on from top to bottom for the purpose of clarity.

Fig. 6 Laser profiles, the IPs and the time-frequency analyses of the HHG for the cases of the combined fields with (a)-(c) $\varphi_{1}=0.2 \pi, \varphi_{1}=1.0 \pi$; (d)-(f) $\varphi_{2}=0.6 \pi, \varphi_{2}=1.0 \pi$; and (g)-(i) $\varphi_{3}=0.2 \pi, \varphi_{3}=1.0 \pi$. Here the CEP of one laser field is varied while those of the other two laser fields are kept zero at each manipulation.

Fig. 7 The HHG spectra driven by the three-color combined fields with (a) $\tau_{\text {delayl }}=0.0$, $0.05 \mathrm{~T}_{800}, 0.15 \mathrm{~T}_{800}, 0.25 \mathrm{~T}_{800} ;$ and (b) $\tau_{\text {delay } 1}=0.0,-0.05 \mathrm{~T}_{800},-0.15 \mathrm{~T}_{800},-0.25 \mathrm{~T}_{800}$. The delay time of $\tau_{\text {delay } 2}$ and the CEPs of the laser fields are $\tau_{\text {delay } 2}=0.0$ and $\varphi_{1}=0.0 \pi, \varphi_{2}=$ $1.0 \pi, \varphi_{3}=0.0 \pi$. The harmonic intensities have been multiplied by factors of $1,10^{-3}, 10^{-6}$, and so on from top to bottom for the purpose of clarity. (c) The original harmonic intensities for the cases of the three-color combined fields with $\tau_{\text {delay } 1}=0.0, \tau_{\text {delay } 2}=0.0$ and $\tau_{\text {delay } 1}=-0.05 \mathrm{~T}_{800}, \tau_{\text {delay } 2}=0.0$.

Fig. 8 Laser profiles and the IPs for the cases of the combined fields with (a) $\tau_{\text {delayl }}=$ $0.15 \mathrm{~T}_{800} ;$ (c) $\tau_{\text {delay } 1}=-0.05 \mathrm{~T}_{800} ;$ (e) $\tau_{\text {delay } 1}=-0.25 \mathrm{~T}_{800}$. The time-frequency analyses of the HHG for the cases of the combined fields with (b) $\tau_{\text {delay } 1}=0.15 \mathrm{~T}_{800} ;(\mathrm{d}) \tau_{\text {delay } 1}=-0.05 \mathrm{~T}_{800}$; 
(f) $\tau_{\text {delay } 1}=-0.25 \mathrm{~T}_{800}$. The delay time of $\tau_{\text {delay } 2}$ and the CEPs of the laser fields are $\tau_{\text {delay } 2}=$ $0.0, \varphi_{1}=0.0 \pi, \varphi_{2}=1.0 \pi$ and $\varphi_{3}=0.0 \pi$.

Fig. 9 The HHG spectra driven by the three-color combined fields with (a) $\tau_{\text {delay } 2}=0.0$, $0.05 \mathrm{~T}_{800}, 0.15 \mathrm{~T}_{800}, 0.25 \mathrm{~T}_{800} ;$ and (b) $\tau_{\text {delay } 2}=0.0,-0.05 \mathrm{~T}_{800},-0.15 \mathrm{~T}_{800},-0.25 \mathrm{~T}_{800}$. The delay time of $\tau_{\text {delay } 1}$ and the CEPs of the laser fields are $\tau_{\text {delay } 1}=-0.05 \mathrm{~T}_{800}, \varphi_{1}=0.0 \pi, \varphi_{2}=$ $1.0 \pi$ and $\varphi_{3}=0.0 \pi$. The harmonic intensities have been multiplied by factors of $1,10^{-3}$, $10^{-6}$, and so on from top to bottom for the purpose of clarity. (c) The original harmonic intensities for the cases of the combined fields with $\tau_{\text {delay } 1}=-0.05 \mathrm{~T}_{800}, \tau_{\text {delay } 2}=0.0$ and $\tau_{\text {delay } 1}=-0.05 \mathrm{~T}_{800}, \tau_{\text {delay } 2}=0.05 \mathrm{~T}_{800}$.

Fig. 10 Laser profiles and the IPs for the cases of the combined fields with (a) $\tau_{\text {delay } 2}=$ $0.05 \mathrm{~T}_{800} ;$ (c) $\tau_{\text {delay } 2}=0.25 \mathrm{~T}_{800} ;$ (e) $\tau_{\text {delay } 2}=-0.25 \mathrm{~T}_{800}$. The time-frequency analyses of the HHG for the cases of the combined fields with (b) $\tau_{\text {delay } 2}=0.05 \mathrm{~T}_{800} ;$ (d) $\tau_{\text {delay } 2}=0.25 \mathrm{~T}_{800}$; (f) $\tau_{\text {delay } 2}=-0.25 \mathrm{~T}_{800}$. The delay time of $\tau_{\text {delay } 1}$ and the CEPs of the laser fields are $\tau_{\text {delay } 1}=$ $-0.05 \mathrm{~T}_{800}, \varphi_{1}=0.0 \pi, \varphi_{2}=1.0 \pi$ and $\varphi_{3}=0.0 \pi$

Fig. 11 The temporal profiles of the SAPs through Fourier transformation of the spectral continuum of the three-color combined fields with (a) $\varphi_{1}=0.0 \pi, \varphi_{2}=1.0 \pi, \varphi_{3}=0.0 \pi$, $\tau_{\text {delay } 1}=0.0, \tau_{\text {delay } 2}=0.0$ (from the total harmonics shown in the inset of Fig. 11(a)), from the $390 \omega_{2000}$ to the $490 \omega_{2000}$, from the $490 \omega_{2000}$ to the $590 \omega_{2000}$, from the $590 \omega_{2000}$ to the $690 \omega_{2000}$ and from the $690 \omega_{2000}$ to the $760 \omega_{2000}$; (b) $\varphi_{1}=0.0 \pi, \varphi_{2}=1.0 \pi, \varphi_{3}=0.0 \pi, \tau_{\text {delay } 1}$ $=-0.05 \mathrm{~T}_{800}, \tau_{\text {delay } 2}=0.0$ from the $400 \omega_{2000}$ to the $500 \omega_{2000}$, from the $500 \omega_{2000}$ to the $600 \omega_{2000}$, from the $600 \omega_{2000}$ to the $700 \omega_{2000}$ and from the $700 \omega_{2000}$ to the $790 \omega_{2000} ;$ (c) $\varphi_{1}$ $=0.0 \pi, \varphi_{2}=1.0 \pi, \varphi_{3}=0.0 \pi, \tau_{\text {delay } 1}=-0.05 \mathrm{~T}_{800}, \tau_{\text {delay } 2}=0.05 \mathrm{~T}_{800}$ from the $380 \omega_{2000}$ to the 
$480 \omega_{2000}$, from the $480 \omega_{2000}$ to the $580 \omega_{2000}$, from the $580 \omega_{2000}$ to the $680 \omega_{2000}$ and from the $680 \omega_{2000}$ to the $780 \omega_{2000}$. The pulses have been shifted in time by $0 \mathrm{fs}, 0.13 \mathrm{fs}, 0.26 \mathrm{fs}$, and $0.39 \mathrm{fs}$ from left to right for the purpose of clarity. 
Table 1 The CEP-dependence $\left(\varphi_{800}\right)$ of the MHCs and the HHCs for the case of the single-color $800 \mathrm{~nm}$ pulse.

\begin{tabular}{cccccccccccc}
\hline$\varphi_{800}(\pi)$ & 0.0 & 0.1 & 0.2 & 0.3 & 0.4 & 0.5 & 0.6 & 0.7 & 0.8 & 0.9 & 1.0 \\
\hline $\operatorname{MHCs}\left(\omega_{800}\right)$ & 81 & 79 & 77 & 75 & 70 & 71 & 74 & 75 & 77 & 79 & 81 \\
\hline $\mathrm{HHCs}\left(\omega_{800}\right)$ & 55 & 59 & 62 & 65 & 70 & 42 & 44 & 47 & 50 & 52 & 55 \\
\hline$\varphi_{800}(\pi)$ & - & 1.1 & 1.2 & 1.3 & 1.4 & 1.5 & 1.6 & 1.7 & 1.8 & 1.9 & 2.0 \\
\hline $\operatorname{MHCs}\left(\omega_{800}\right)$ & - & 79 & 77 & 75 & 70 & 71 & 74 & 75 & 77 & 79 & 81 \\
\hline $\mathrm{HHCs}\left(\omega_{800}\right)$ & - & 59 & 62 & 65 & 70 & 42 & 44 & 47 & 50 & 52 & 55 \\
\hline
\end{tabular}


Table 2 The CEP-dependence $\left(\varphi_{2000}\right)$ of the MHCs and the HHCs for the case of the single-color $2000 \mathrm{~nm}$ pulse.

\begin{tabular}{ccccccccccccc}
\hline$\varphi_{2000}(\pi)$ & 0.0 & 0.1 & 0.2 & 0.3 & 0.4 & 0.5 & 0.6 & 0.7 & 0.8 & 0.9 & 1.0 \\
\hline $\operatorname{MHCs}\left(\omega_{2000}\right)$ & 137 & 133 & 135 & 135 & 133 & 135 & 133 & 133 & 131 & 131 & 133 \\
\hline $\operatorname{HHCs}\left(\omega_{2000}\right)$ & 135 & 133 & 135 & 135 & 133 & 133 & 131 & 133 & 133 & 131 & 131 \\
\hline$\varphi_{2000}(\pi)$ & - & 1.1 & 1.2 & 1.3 & 1.4 & 1.5 & 1.6 & 1.7 & 1.8 & 1.9 & 2.0 \\
\hline $\operatorname{MHCs}\left(\omega_{2000}\right)$ & - & 133 & 135 & 135 & 133 & 135 & 133 & 133 & 131 & 131 & 133 \\
\hline $\operatorname{HHCs}\left(\omega_{2000}\right)$ & - & 133 & 135 & 135 & 133 & 133 & 131 & 133 & 133 & 131 & 131 \\
\hline
\end{tabular}


Table 3 The CEP-dependence $\left(\varphi_{2000}\right)$ of the MHCs and the HHCs for the case of the two-color field. The CEP of $\varphi_{800}$ is chosen to be $\varphi_{800}=0.0 \pi$.

\begin{tabular}{ccccccccccccc}
\hline$\varphi_{2000}(\pi)$ & 0.0 & 0.1 & 0.2 & 0.3 & 0.4 & 0.5 & 0.6 & 0.7 & 0.8 & 0.9 & 1.0 \\
\hline $\operatorname{MHCs}\left(\omega_{2000}\right)$ & 403 & 383 & 357 & 303 & 257 & 288 & 301 & 293 & 318 & 311 & 287 \\
\hline $\operatorname{HHCs}\left(\omega_{2000}\right)$ & 152 & 165 & 179 & 217 & 257 & 115 & 123 & 130 & 126 & 120 & 200 \\
\hline$\varphi_{2000}(\pi)$ & - & 1.1 & 1.2 & 1.3 & 1.4 & 1.5 & 1.6 & 1.7 & 1.8 & 1.9 & 2.0 \\
\hline $\operatorname{MHCs}\left(\omega_{2000}\right)$ & - & 247 & 178 & 193 & 208 & 202 & 243 & 197 & 347 & 282 & 403 \\
\hline $\operatorname{HHCs}\left(\omega_{2000}\right)$ & - & 163 & 159 & 193 & 208 & 201 & 243 & 112 & 125 & 143 & 152 \\
\hline
\end{tabular}




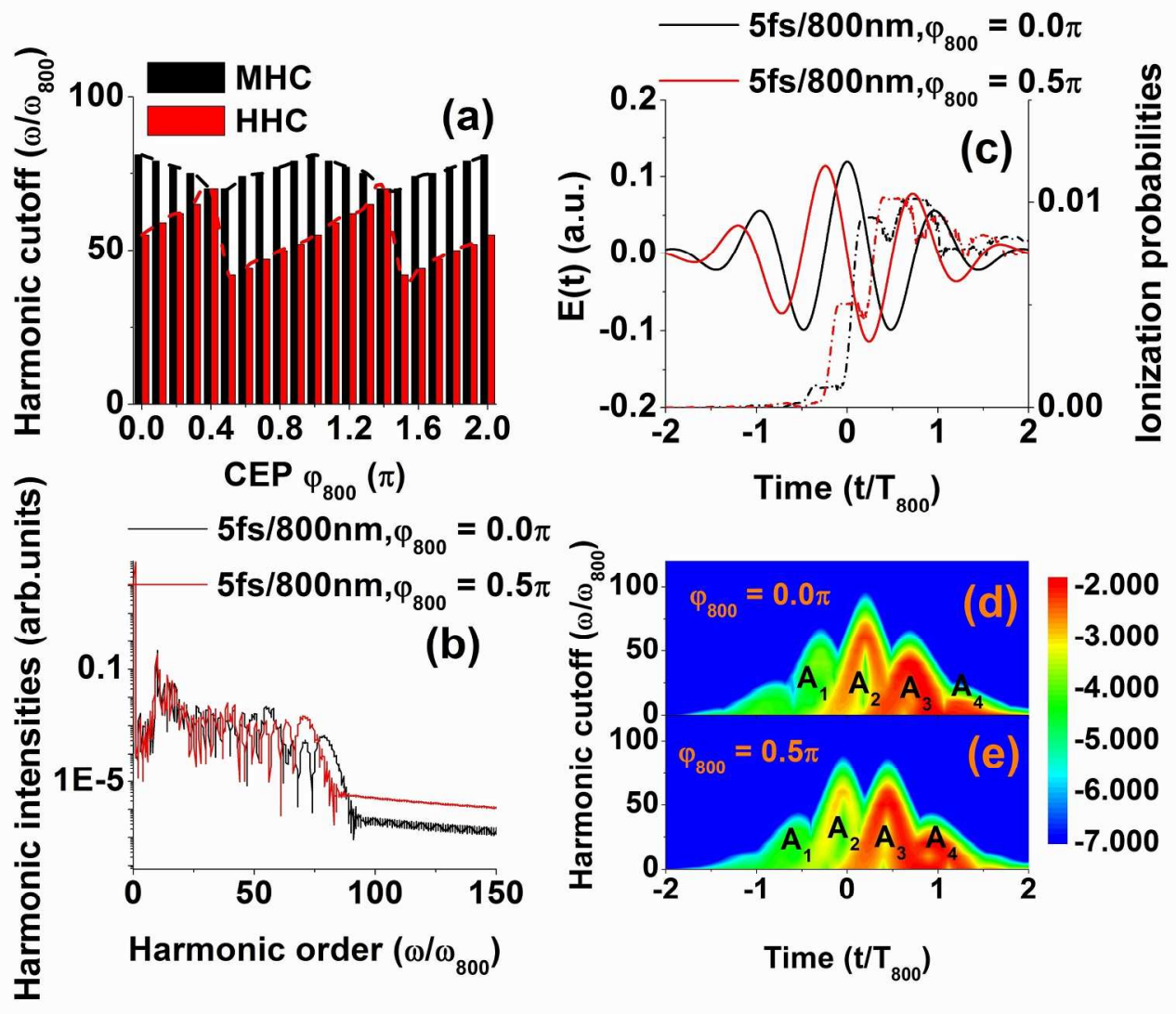

Figure 1 (a) The CEP dependence of the MHCs and the HHCs driven by the single-color 800nm field. The FWHM and the laser intensity is $5 \mathrm{fs}$ and $1800=5.0 \times 1014 \mathrm{~W} / \mathrm{cm} 2$. (b) The HHG spectra from the above single-color $5 \mathrm{fs} / 800 \mathrm{~nm}$ fields with $\varphi 800=0.0 \pi$ and $\varphi 800=0.5 \pi$. (c) Laser profiles and the IPs of the single-color $5 \mathrm{fs} / 800 \mathrm{~nm}$ fields with $\varphi 800=0.0 \pi$ and $\varphi 800=0.5 n$. The time-frequency analyses of the HHG for the cases of the single-color $5 \mathrm{fs} / 800 \mathrm{~nm}$ fields with (d) $\varphi 800=0.0 \pi$ and (e) $\varphi 800=0.5 n$. T800 is the optical cycle of the $800 \mathrm{~nm}$ pulse. 

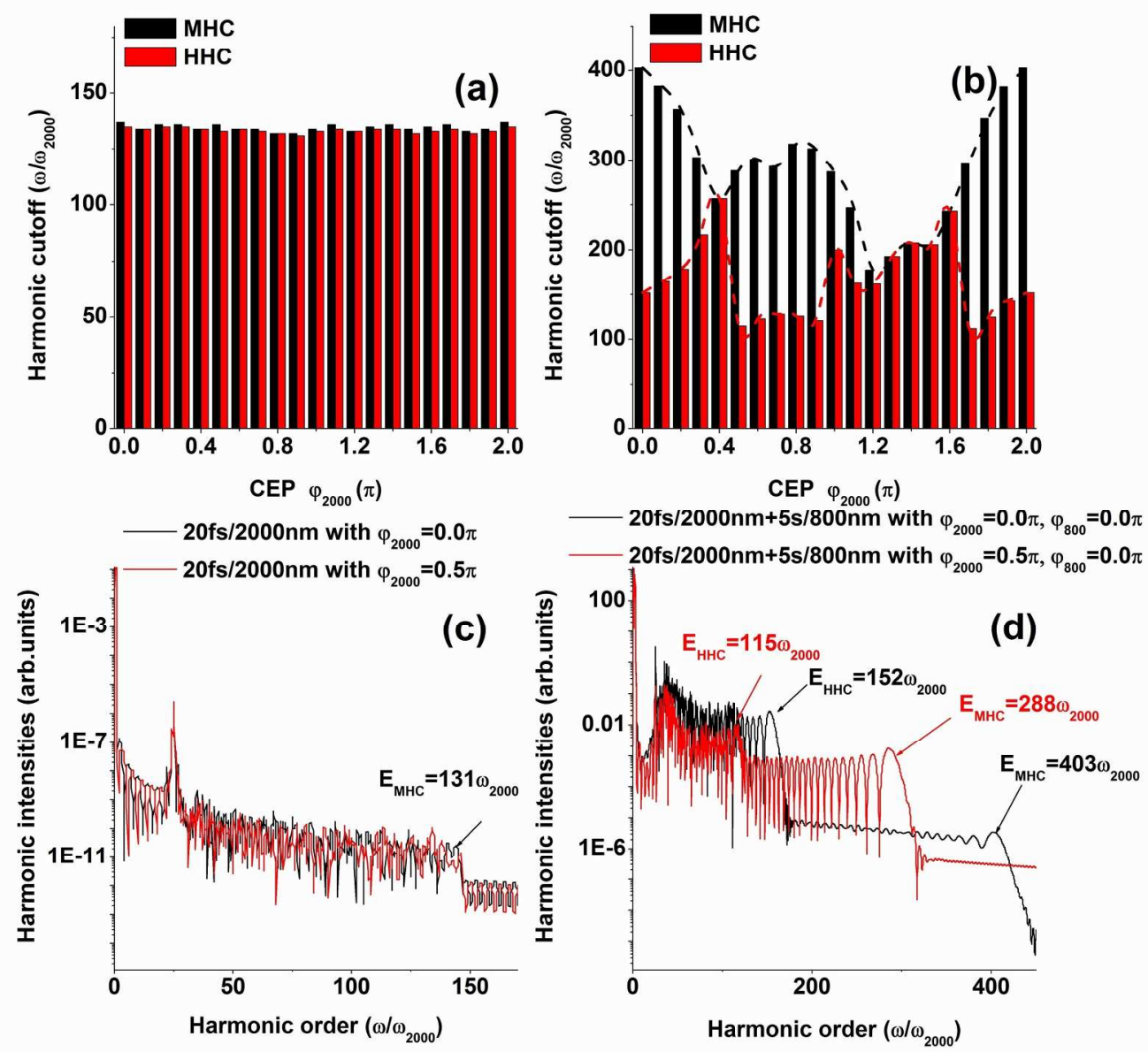

Figure 2 The CEP dependence of the MHCs and the HHCs driven by (a) the single-color $20 \mathrm{fs} / 2000 \mathrm{~nm}$ field and (b) the combined field consisting of the $20 \mathrm{fs} / 2000 \mathrm{~nm}$ field and the $5 \mathrm{fs} / 800 \mathrm{~nm}$ field with tdelay $1=$ $0.0, \varphi 800=0.0$. The pulse intensities are $\mathrm{I} 2000=5.0 \times 1013 \mathrm{~W} / \mathrm{cm} 2$ and $\mathrm{I} 800=5.0 \times 1014 \mathrm{~W} / \mathrm{cm} 2$, respectively. The HHG spectra from (c) the single-color $20 \mathrm{fs} / 2000 \mathrm{~nm}$ fields with $\varphi 2000=0.0 \Pi$ and $\varphi 2000$ $=0.5 n$; and (d) the combined fields with $\varphi 2000=0.0 n, \varphi 800=0.0 n$ and $\varphi 2000=0.5 n, \varphi 800=0.0 \pi$.

$$
661 \times 661 \mathrm{~mm}(96 \times 96 \mathrm{DPI})
$$




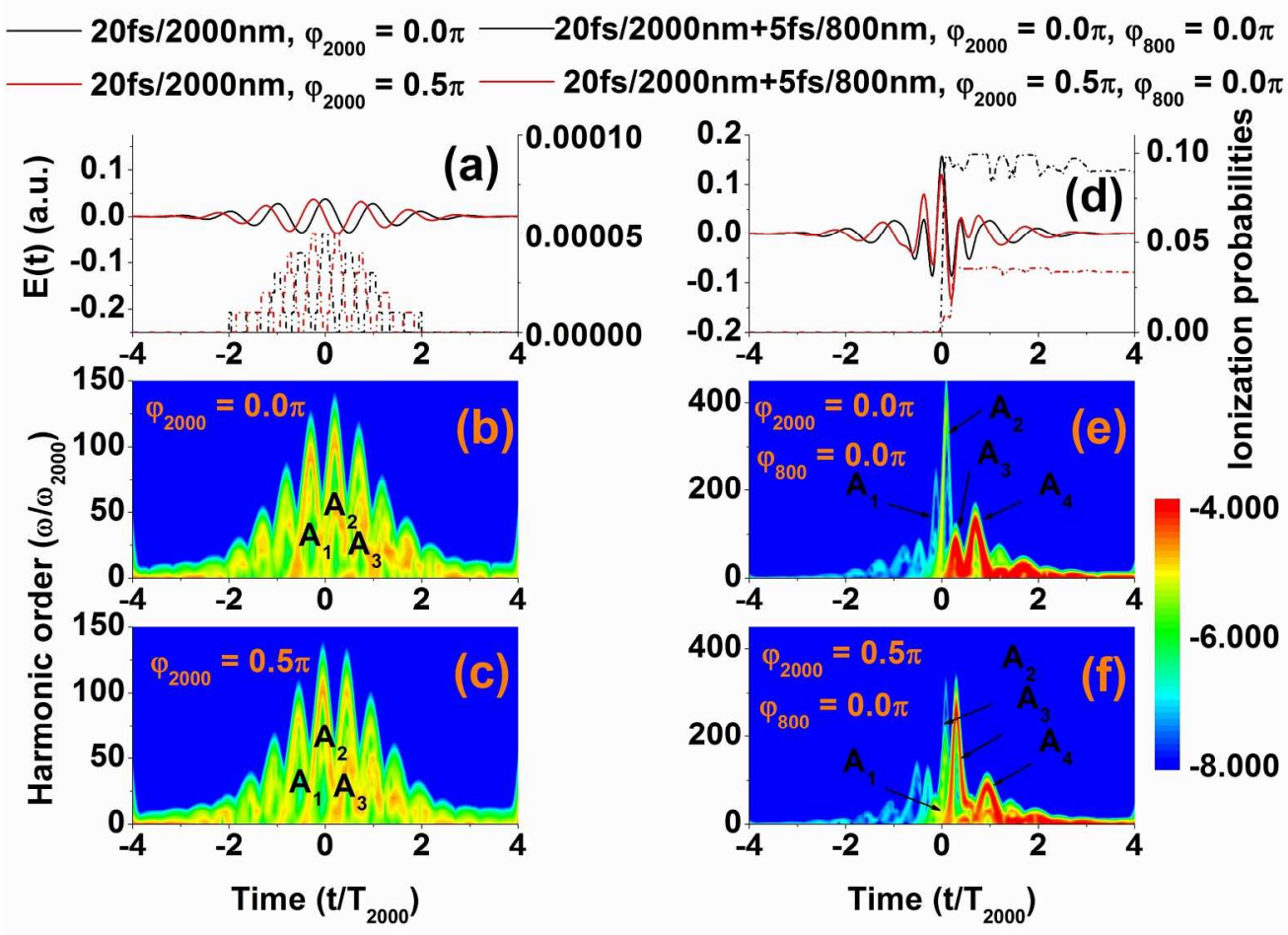

Figure 3 Laser profiles of (a) the single-color $20 \mathrm{fs} / 2000 \mathrm{~nm}$ fields with $\varphi 2000=0.0 n$ and $\varphi 2000=0.5 n$ and (d) the combined fields with $\varphi 2000=0.0 \pi, \varphi 800=0.0 \Pi$ and $\varphi 2000=0.5 \pi, \varphi 800=0.0 \pi$, respectively.

The time-frequency analyses of the HHG for the cases of the single-color $20 \mathrm{fs} / 2000 \mathrm{~nm}$ fields with (b) $\varphi 2000=0.0 \pi$ and (c) $\varphi 2000=0.5 \Pi$ and the combined fields with (e) $\varphi 2000=0.0 \pi, \varphi 800=0.0 \pi$ and (f) $\varphi 2000=0.5 n, \varphi 800=0.0 n$. T2000 means the optical cycle of the $2000 \mathrm{~nm}$ pulse.

$661 \times 506 \mathrm{~mm}(96 \times 96 \mathrm{DPI})$ 

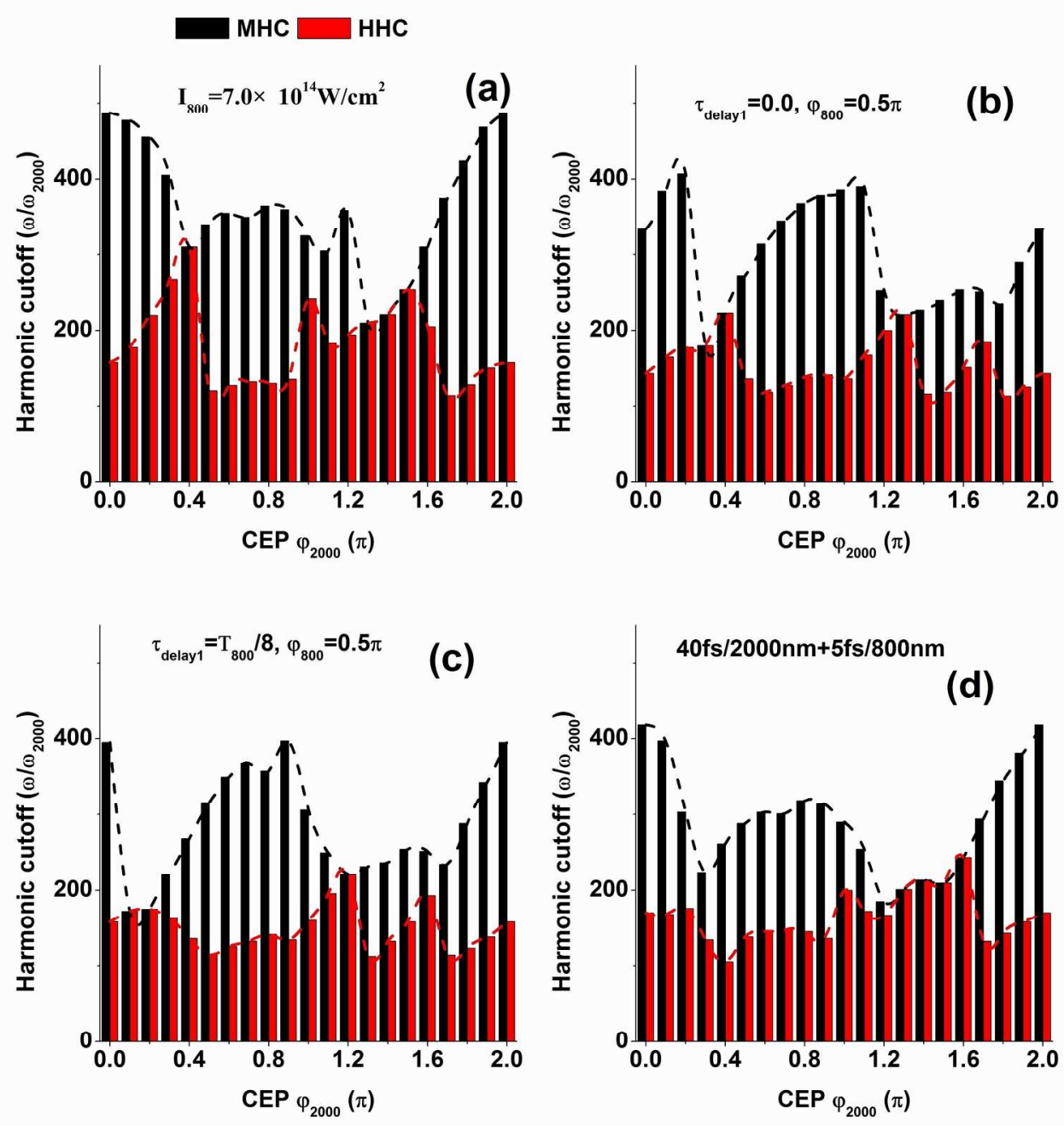

Figure 4 The CEP dependence of the MHCs and the HHCs of the two-color combined field with (a) I800 $=$ $7.0 \times 1014 \mathrm{~W} / \mathrm{cm} 2$; (b) Tdelay1 $=0.0, \varphi 800=0.5 \Pi$; (c) Tdelay $1=\mathrm{T} 800 / 8, \varphi 800=0.5 \Pi$; (d) $\mathrm{T} 2000=40 \mathrm{fs}$. The other parameters are the same as those in Fig. 2(b) except for the above given parameters. 


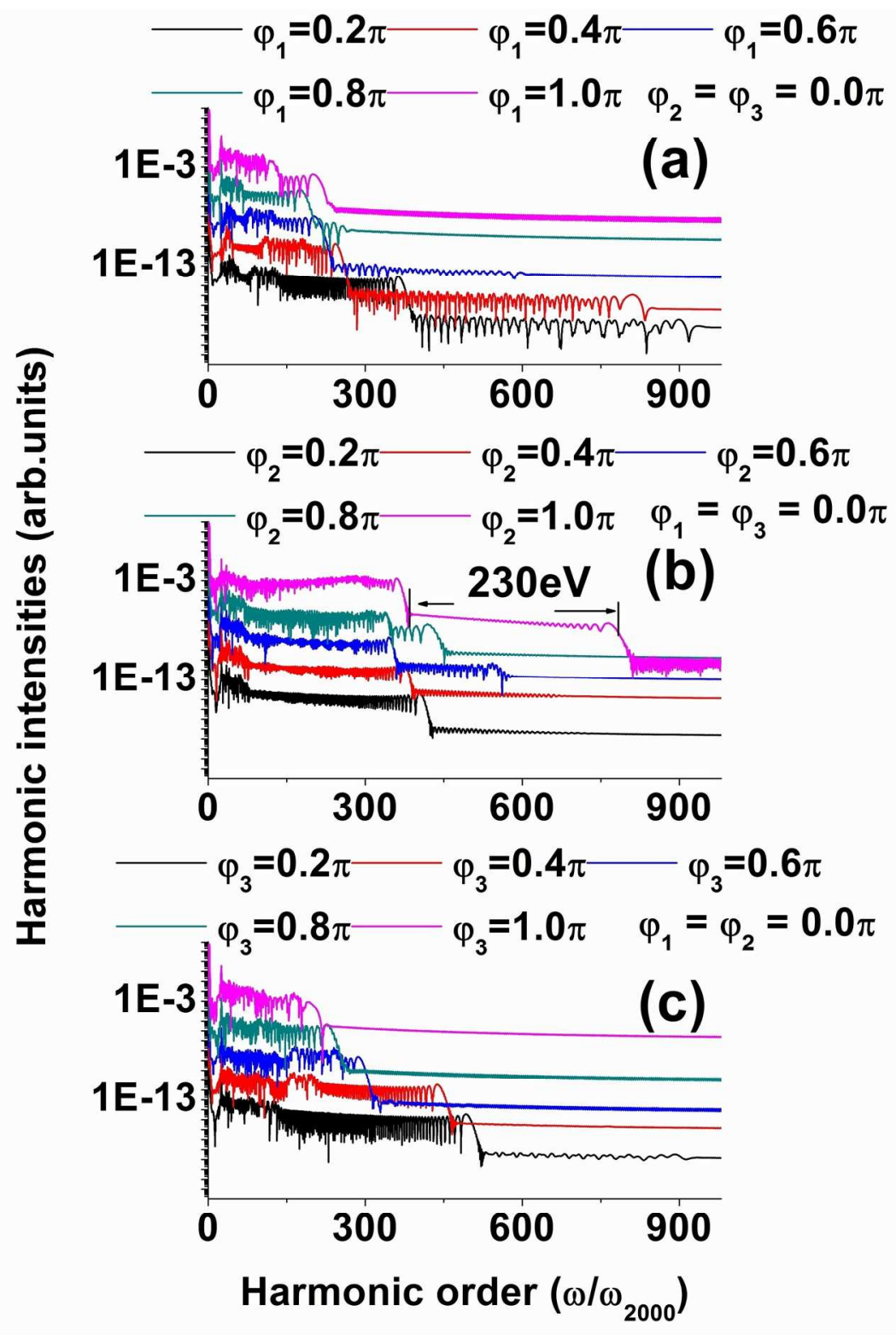

Figure 5 The HHG spectra driven by the three-color combined fields with different CEPs (a) $\varphi 1$; (b) $\varphi 2$; and

(c) $\varphi 3$. Here the CEP of one laser field is varied while those of the other two laser fields are kept zero at each manipulation. The laser fields are chosen to be $20 \mathrm{fs} / 2000 \mathrm{~nm}, \mathrm{I} 1=5.0 \times 1013 \mathrm{~W} / \mathrm{cm} 2,5 \mathrm{fs} / 800 \mathrm{~nm}, \mathrm{I} 2$ $=5.0 \times 1014 \mathrm{~W} / \mathrm{cm} 2$ and $20 \mathrm{fs} / 2400 \mathrm{~nm}, \mathrm{I} 3=5.0 \times 1013 \mathrm{~W} / \mathrm{cm} 2$. The harmonic intensities have been multiplied by factors of $1,10-3,10-6$, and so on from top to bottom for the purpose of clarity.

$$
444 \times 661 \mathrm{~mm}(96 \times 96 \mathrm{DPI})
$$



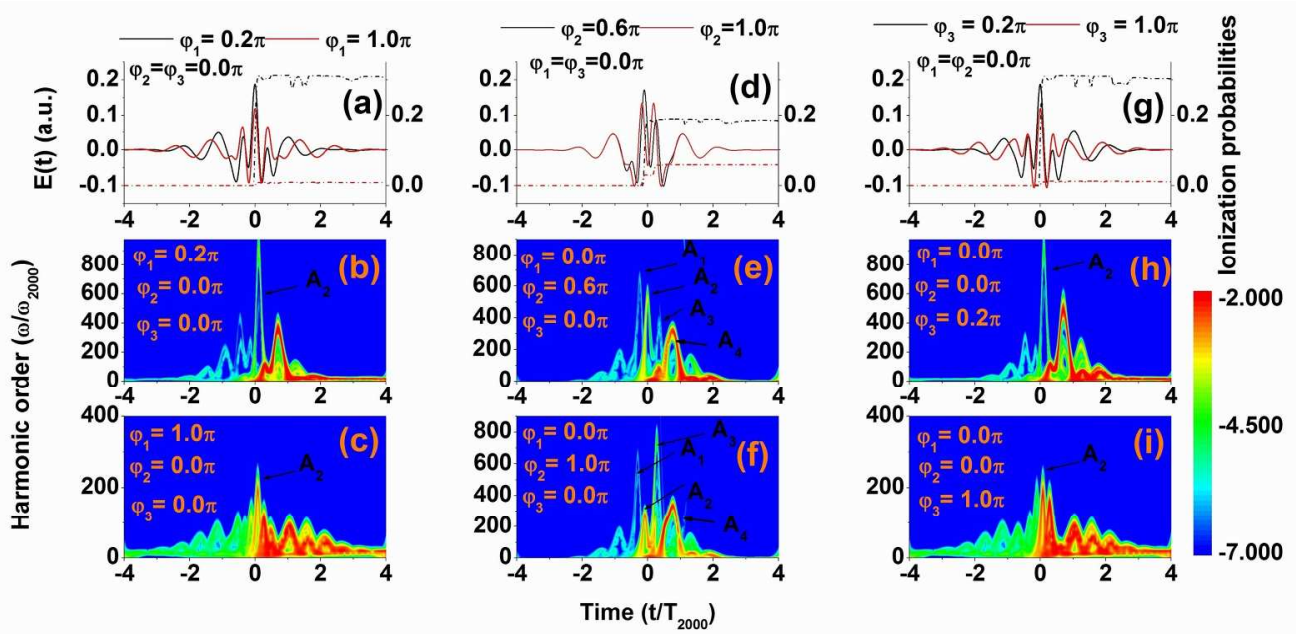

Figure 6 Laser profiles, the IPs and the time-frequency analyses of the HHG for the cases of the combined fields with (a)-(c) $\varphi 1=0.2 \pi, \varphi 1=1.0 \pi$; (d)-(f) $\varphi 2=0.6 \pi, \varphi 2=1.0 \pi$; and (g)-(i) $\varphi 3=0.2 \pi, \varphi 3=1.0 \pi$. Here the CEP of one laser field is varied while those of the other two laser fields are kept zero at each manipulation.

$661 \times 327 \mathrm{~mm}(96 \times 96 \mathrm{DPI})$ 


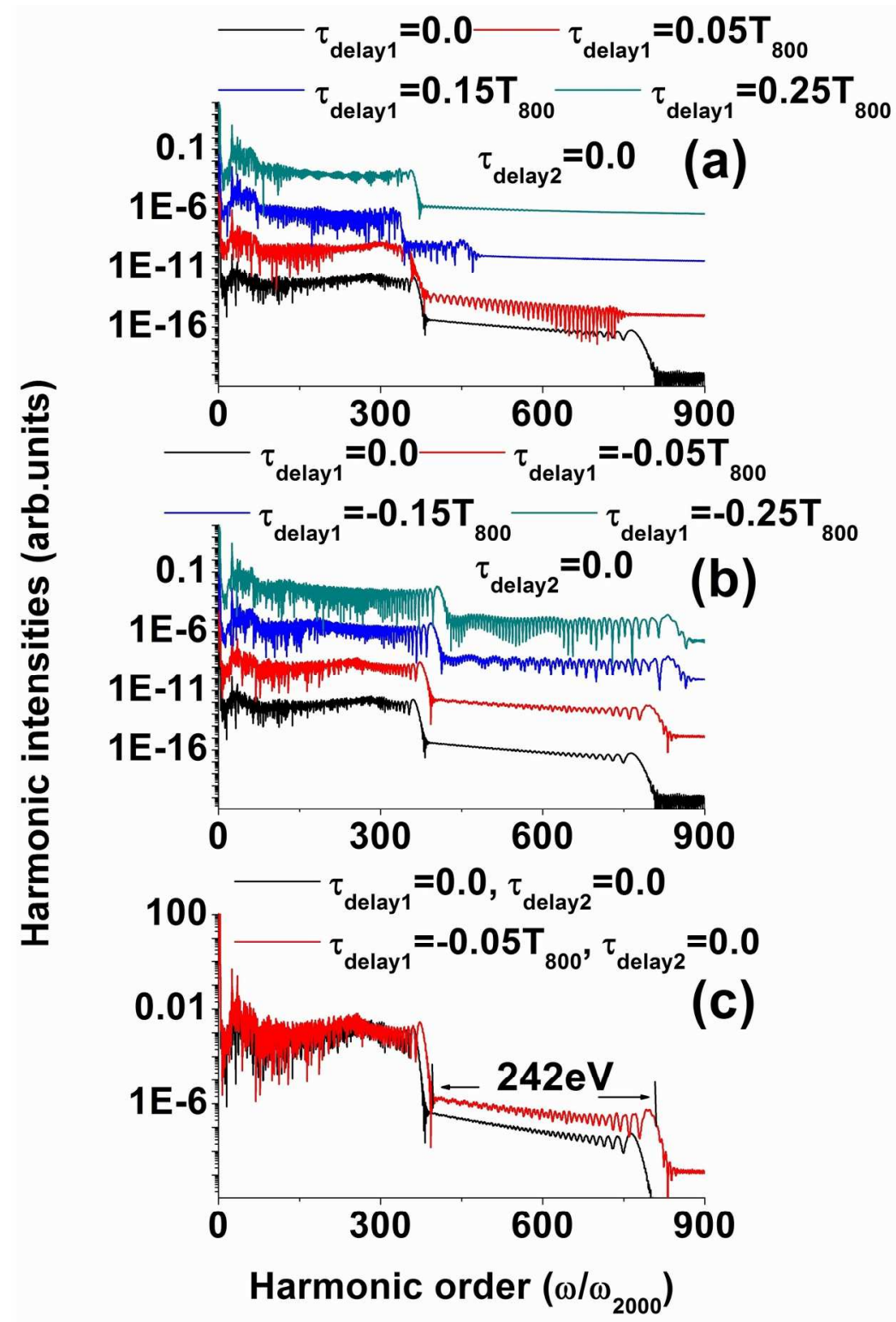

Figure 7 The HHG spectra driven by the three-color combined fields with (a) Tdelay1 $=0.0,0.05$ T800, 0.15 T800, 0.25T800; and (b) Tdelay $1=0.0,-0.05 T 800,-0.15 T 800,-0.25 T 800$. The delay time of tdelay 2 and the CEPs of the laser fields are tdelay $2=0.0$ and $\varphi 1=0.0 \pi, \varphi 2=1.0 \pi, \varphi 3=0.0 \pi$. The harmonic intensities have been multiplied by factors of $1,10-3,10-6$, and so on from top to bottom for the purpose of clarity. (c) The original harmonic intensities for the cases of the three-color combined fields with tdelay $1=$ 0.0 , tdelay $2=0.0$ and tdelay $1=-0.05 T 800$, tdelay $2=0.0$. 


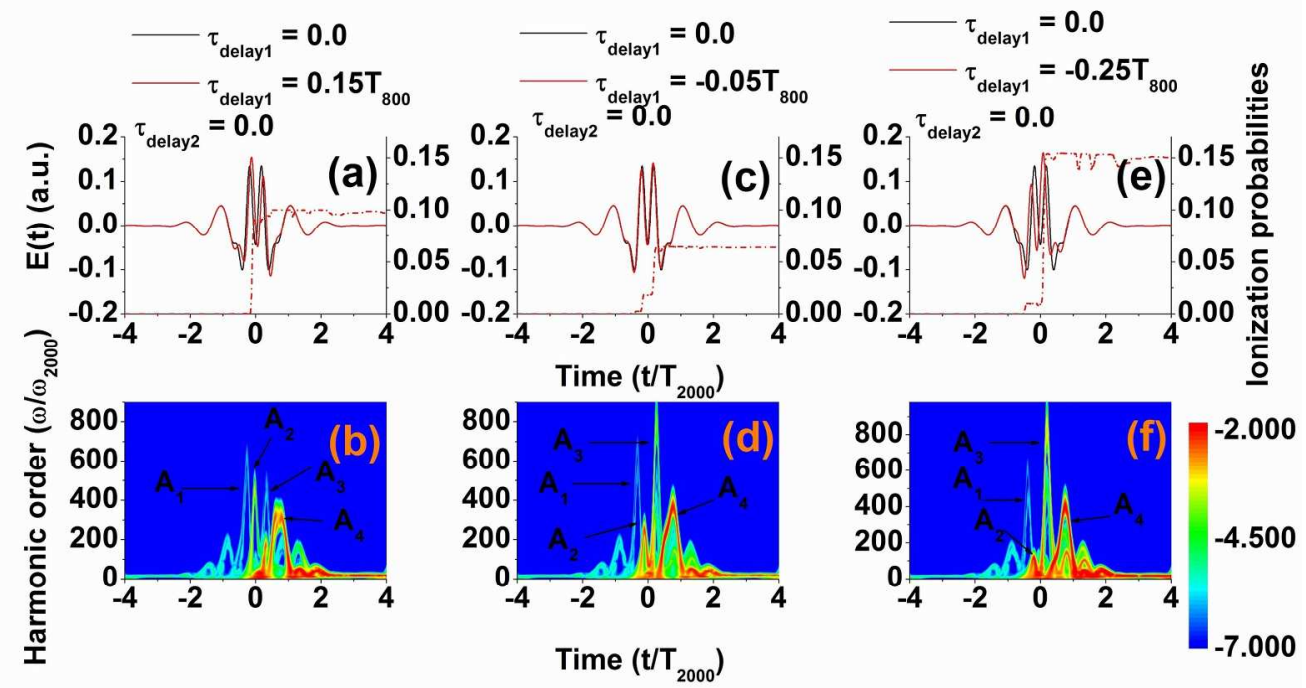

Figure 8 Laser profiles and the IPs for the cases of the combined fields with (a) Tdelay $1=0.15 T 800$; (c) Tdelay1 $=-0.05$ T800; (e) Tdelay1 $=-0.25 T 800$. The time-frequency analyses of the HHG for the cases of the combined fields with $(b)$ Tdelay $1=0.15 T 800 ;(d)$ Tdelay $1=-0.05 T 800 ;(f)$ Tdelay $1=-0.25 T 800$. The delay time of tdelay 2 and the CEPs of the laser fields are tdelay $2=0.0, \varphi 1=0.0 n, \varphi 2=1.0 n$ and $\varphi 3=$ 0.0 .

$661 \times 363 \mathrm{~mm}(96 \times 96 \mathrm{DPI})$ 


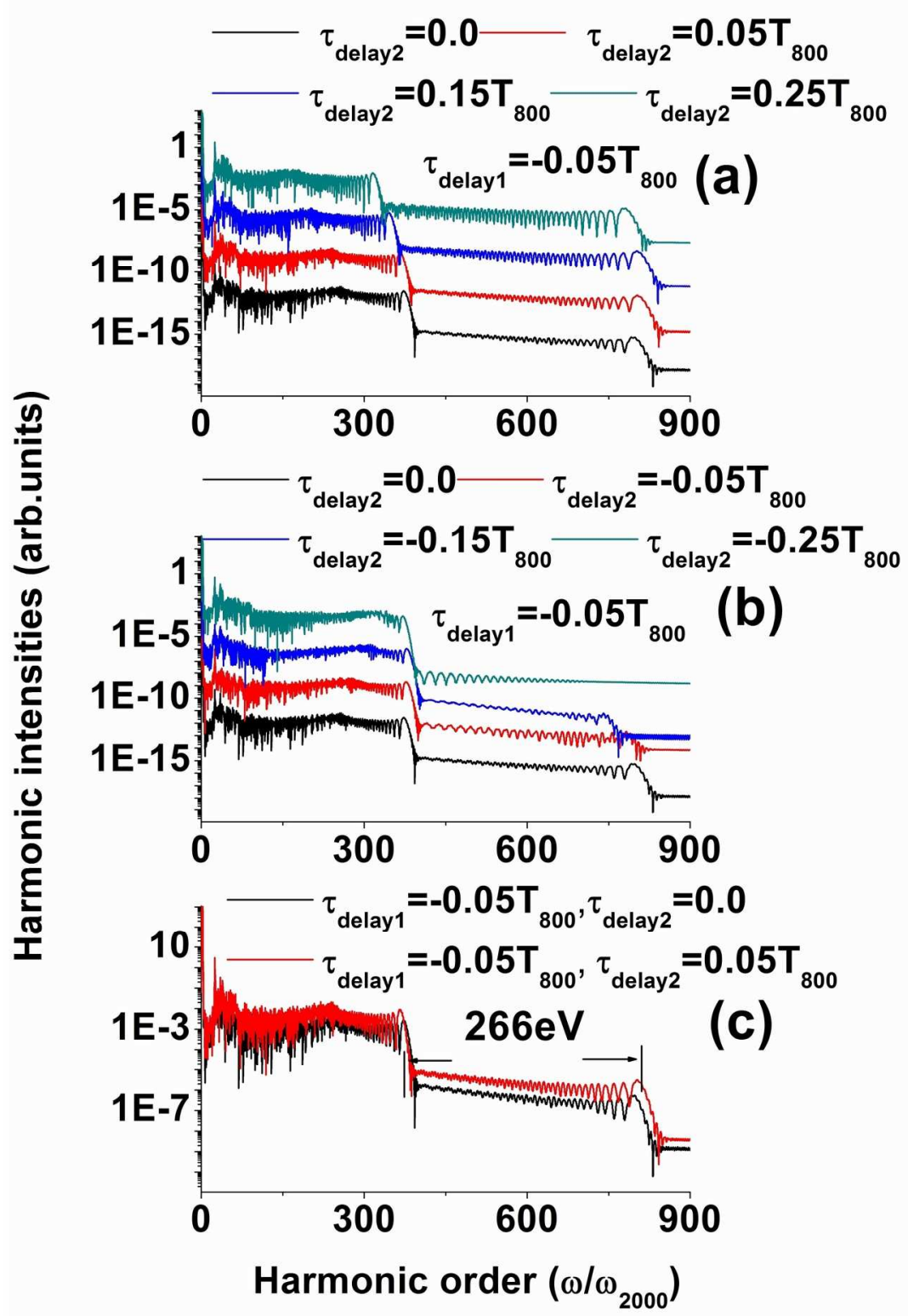

Figure 9 The HHG spectra driven by the three-color combined fields with (a) Tdelay2 $=0.0,0.05$ T800, 0.15 T800, 0.25T800; and (b) Tdelay $2=0.0,-0.05 T 800,-0.15 T 800,-0.25 T 800$. The delay time of tdelay 1 and the CEPs of the laser fields are tdelay $1=-0.05 T 800, \varphi 1=0.0 \pi, \varphi 2=1.0 \pi$ and $\varphi 3=0.0 \pi$. The harmonic intensities have been multiplied by factors of $1,10-3,10-6$, and so on from top to bottom for the purpose of clarity. (c) The original harmonic intensities for the cases of the combined fields with tdelay $1=-$ 0.05 T 800 , tdelay $2=0.0$ and tdelay $1=-0.05$ T 800 , tdelay $2=0.05$ T 800 .

$456 \times 661 \mathrm{~mm}(96 \times 96$ DPI $)$ 


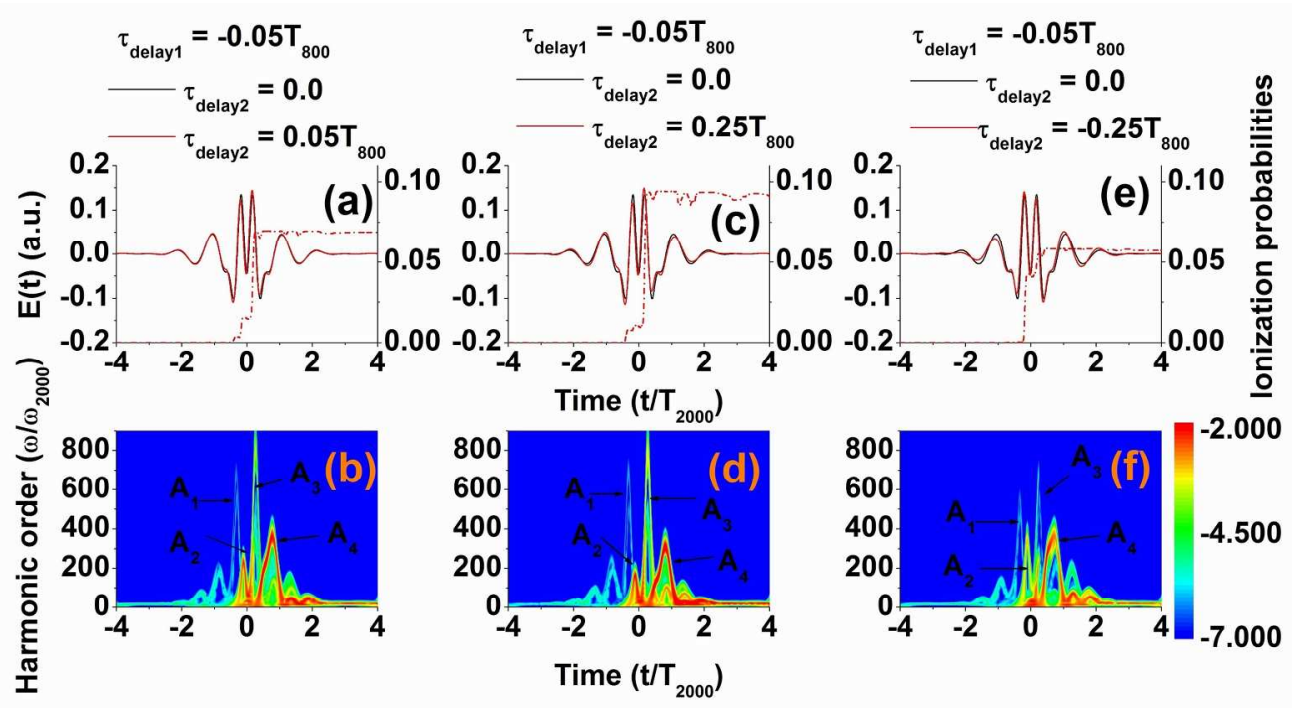

Figure 10 Laser profiles and the IPs for the cases of the combined fields with (a) Tdelay2 $=0.05$ T800; (c) Tdelay $2=0.25 \mathrm{~T} 800 ;(\mathrm{e})$ Tdelay $2=-0.25 \mathrm{~T} 800$. The time-frequency analyses of the HHG for the cases of the combined fields with (b) Tdelay $2=0.05 T 800 ;(d)$ Tdelay $2=0.25 T 800 ;(f)$ tdelay $2=-0.25$ T 800 . The delay time of Tdelay 1 and the CEPs of the laser fields are tdelay $1=-0.05 T 800, \varphi 1=0.0 \Pi, \varphi 2=1.0 \Pi$ and $\varphi 3=$ 0.0 . 


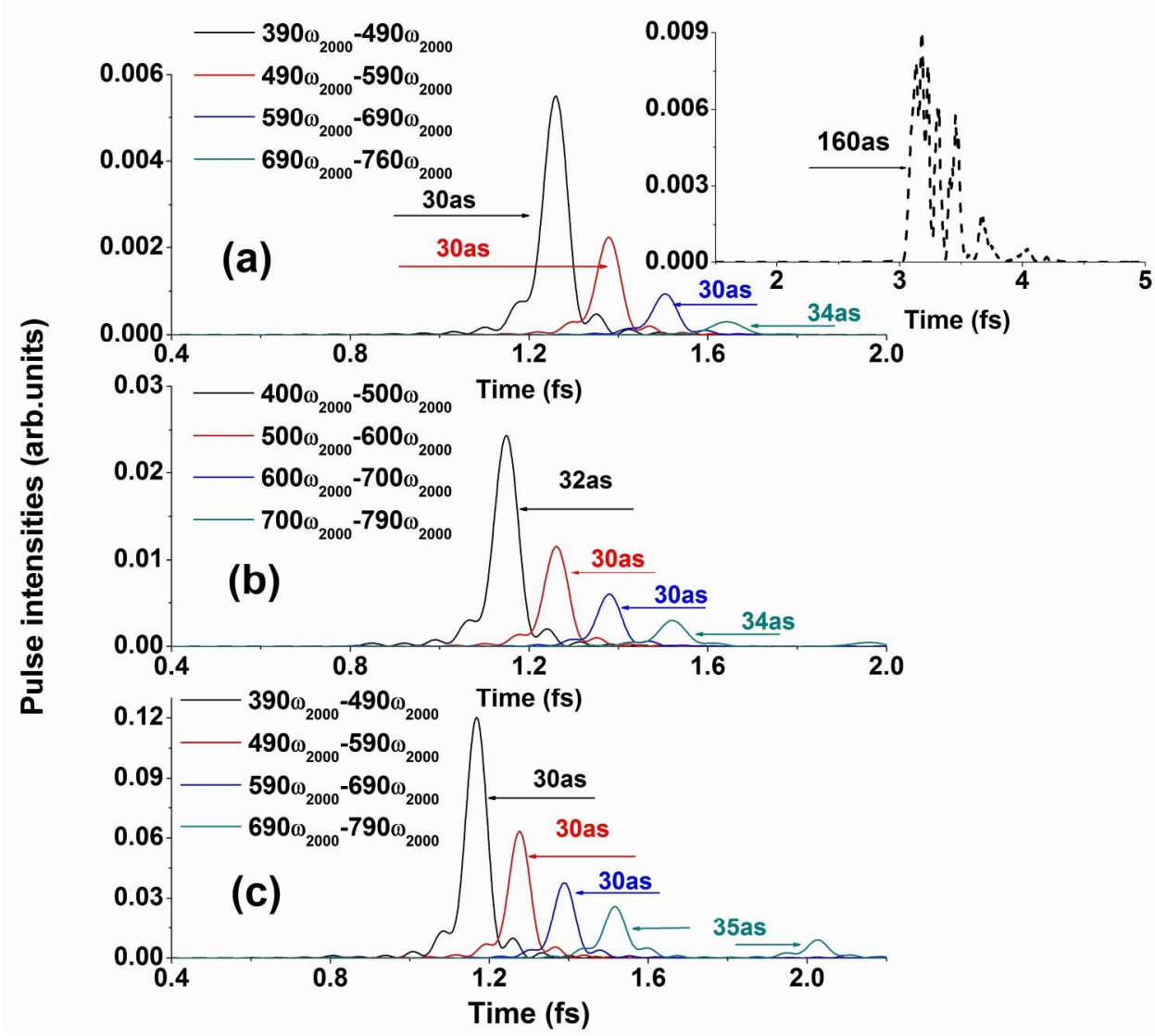

Figure 11 The temporal profiles of the SAPs through Fourier transformation of the spectral continuum of the three-color combined fields with (a) $\varphi 1=0.0 \pi, \varphi 2=1.0 n, \varphi 3=0.0 \pi$, tdelay1 $=0.0$, tdelay2 $=0.0$ (from the total harmonics shown in the inset of Fig. 11(a)), from the $390 \omega 2000$ to the $490 \omega 2000$, from the $490 \omega 2000$ to the $590 \omega 2000$, from the $590 \omega 2000$ to the $690 \omega 2000$ and from the $690 \omega 2000$ to the $760 \omega 2000$; (b) $\varphi 1=0.0 \pi, \varphi 2=1.0 \pi, \varphi 3=0.0 \pi$, tdelay $1=-0.05 T 800$, tdelay $2=0.0$ from the $400 \omega 2000$ to the $500 \omega 2000$, from the $500 \omega 2000$ to the $600 \omega 2000$, from the $600 \omega 2000$ to the $700 \omega 2000$ and from the

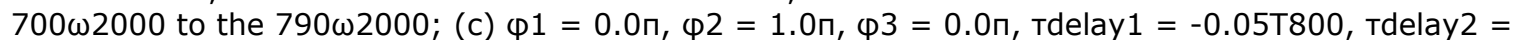
0.05 T800 from the $380 \omega 2000$ to the $480 \omega 2000$, from the $480 \omega 2000$ to the $580 \omega 2000$, from the $580 \omega 2000$ to the $680 \omega 2000$ and from the $680 \omega 2000$ to the $780 \omega 2000$. The pulses have been shifted in time by 0 fs, $0.13 \mathrm{fs}, 0.26 \mathrm{fs}$, and $0.39 \mathrm{fs}$ from left to right for the purpose of clarity.

$661 \times 569 \mathrm{~mm}(96 \times 96 \mathrm{DPI})$ 